\title{
Konsumausgaben als Wohlstandsindikator - was können sie uns über Armut und Ungleichheit berichten?
}

\section{Consumption as an Indicator of Well-being - What Can It Teach Us about Poverty and Inequality?}

\author{
Ein Vergleich einkommens- und konsumbasierter Armuts- und Ungleichheitsmaße mit Hilfe \\ der Einkommens- und Verbrauchsstichprobe \\ A Comparison of Income- and Consumption-Based Poverty and Inequality Measures Based on \\ the German Income and Expenditure Survey
}

\section{DOI 10.1515/zfsoz-2015-1011}

Zusammenfassung: In der internationalen Forschung gilt der Konsum als adäquater Indikator für den Wohlstand eines Haushalts, in der deutschsprachigen Forschung hingegen das Einkommen. Der vorliegende Artikel untersucht, inwiefern sich konsum- und einkommensbasierte Wohlstandsindikatoren unterscheiden und ob durch die Berücksichtigung der Konsumausgaben eine Gruppe hinsichtlich verschiedener Lebensstandardmerkmale benachteiligter Haushalte identifiziert werden kann. Die Ergebnisse zeigen, dass sich auf der Basis der Konsumausgaben ein positiveres Urteil für Deutschland bezüglich Ungleichheit und Armut fällen lässt als auf der Basis der Einkommen. Die Betrachtung verschiedener Lebensstandardmerkmale zeigt außerdem, dass durch die Konsumperspektive eine Gruppe von Haushalten in den Fokus der Armutsanalysen rückt, die hoch verschuldet ist und der nur ein geringer Anteil ihrer Ausgaben zur Wahrung sozialer Teilhabe verbleibt.

Schlüsselwörter: Konsum; Armut; Ungleichheit; Lebensstandard; Einkommens- und Verbrauchsstichprobe.

Abstract: Whereas international studies discuss consumption expenditures as an adequate indicator of the well-being of households and individuals, social and economic analyses in Germany mainly focus on income. This article uses data from the German Income and Ex-

*Korrespondenzautor: Katharina Hörstermann, Institut für Soziologie und Sozialpsychologie, Universität zu Köln, Albertus-MagnusPlatz, 50923 Köln, E-Mail: hoerstermann@wiso.uni-koeln.de penditure Survey to examine how income- and consumption-based measures differ. Furthermore, it compares the standard of living of households which have low income with those which have low expenditures. Of particular interest are households that are defined as poor by expenditures but not by income. The results indicate that the consumption-based measures draw a less negative picture of poverty and inequality in Germany. On the other hand, the description of the standard of living reveals that consumption-based analyses point out a group of households with high indebtedness and low spending in categories of social participation.

Keywords: Consumption; Poverty; Inequality; Standard of Living; Income and Expenditure Survey.

\section{Einleitung}

Sozialpolitiker sprechen gerne über Armut und Ungleichheit. Im Allgemeinen haben ihre Äußerungen den gleichen Tenor: Deutschland leide unter einer steigenden Ungleichverteilung der Einkommen und zunehmender Armut. Dabei beziehen sie sich auf Studien wie z. B. die des Deutschen Paritätischen Wohlfahrtsverbands (2015), der in seinem Armutsbericht Deutschland als eine „,zerklüftete Republik“ tituliert, in der die Armut einen historischen Höchststand erreicht habe und sich deutliche regionale Differenzen zeigen. Dabei sei seitens der Bundesregierung keine politische Weichenstellung erkennbar, die Hoffnung auf eine Umkehr dieses Trends erkennen ließe. Als Beleg 
präsentiert der Verband Berechnungen von Armutsquoten, die diejenigen Personen als arm definieren, denen weniger als $60 \%$ des bedarfsgewichteten Medianeinkommens zur Verfügung steht. Diese Definition von Armut in Relation zum jeweiligen sozialen Umfeld ist in der sozialpolitischen Forschung durchaus üblich und wird beispielweise auch von der WHO, der OECD oder der EU verwen$\operatorname{det}^{1}$.

Kritiker weisen allerdings darauf hin, dass das Einkommen eines Haushalts nicht zwangsläufig etwas über den tatsächlichen Lebensstandard aussagt. Einkommensressourcen werden erst im Haushaltskontext in Wohlstand umgewandelt und dabei von Bedarfslagen (z. B. Krankheit), der Effektivität der Ressourcenverwendung und zusätzlichen nicht-monetären Ressourcen (z. B. sozialen Netzwerken) beeinflusst (Groh-Samberg \& Goebel 2007). Durch einen effizienten Einsatz des verfügbaren Einkommens können somit auch einkommensarme Personen einen akzeptablen Lebensstandard realisieren. Zudem ist die Vorrangstellung der einkommensbasierten Armutsmaße weniger auf wissenschaftliche oder methodische Überzeugung zurückzuführen als auf pragmatische Einübung (Klocke 2000).

In der Armutsforschung wird daher in den letzten Jahrzehnten verstärkt eine konzeptionell umfassendere Abbildung von Armut gefordert (Berner 2015). Neben dem Einkommen sollen dabei auch materielle Ausstattungsmerkmale und die Chancen der sozialen Teilhabe Berücksichtigung finden, um den Zusammenhang der ökonomischen Armutslage mit anderen Lebensbereichen gehaltvoll erschließen zu können (Klocke 2000). Mit dem Lebenslagen- und Deprivationsansatz wurden daher auch zwei Konzepte in das Repertoire der Armutsforschung aufgenommen, die Armut als multidimensionales Problem betrachten und ihren Fokus auf die Unterschreitung von Mindeststandards bzw. Unterversorgung in zentralen Lebenslagendimensionen richten, wie z.B. Bildung, Wohnsituation, Gesundheit und soziale Einbindung (vgl. Neurath 1981; Townsend 1979).

Im internationalen Kontext wird ein weiterer Wohlstandsindikator diskutiert, dem in Deutschland noch wenig Beachtung geschenkt wird: Die Konsumausgaben bzw. die Art der Einkommensverwendung. Wie im Falle der relativen Einkommensarmut steht auch bei diesem Ansatz die Verteilung einer monetären Ressource im Zentrum des

1 Bei dem Konzept der relativen Einkommensarmut herrscht allerdings keine Einigkeit über die Höhe der Armutsschwelle. Während die EU diese beispielsweise bei $60 \%$ des bedarfsgewichteten Medianeinkommens ansetzt, haben sich die OECD und die WHO für die $50 \%$-Grenze entschieden.
Interesses, und viele der methodischen Probleme (z. B. die Bestimmung der Armutsgrenze) finden sich wieder. Befürworter der konsumbasierten Wohlstandsmessung argumentieren jedoch, dass der Konsum den Wohlstand adäquater erfassen könne und sich sozialpolitische Entscheidungsträger bei der Bestimmung von Sozialleistungen und der Evaluation von Armutsprogrammen daher nicht nur am Einkommen orientieren sollten (vgl. Meyer \& Sullivan 2003). Die Ausgaben und deren Entwicklung werden als Ausdruck des Lebensstandards von Individuen, Haushalten und der Gesellschaft wahrgenommen und dienen zusätzlich als Maß zur Beschreibung und Abschätzung der Partizipation von Haushalten an der Wohlfahrt der Gesellschaft (Bögenhold \& Fachinger 2000). Bögenhold und Fachinger (2005: 10) bezeichnen daher die Einkommensverwendung als „eine Seite der Münze des sozialen Agierens von Personen, die zu wenig beachtet wurde.“ Sie fordern, dass die Evaluation sozialpolitischer Merkmale auch Muster des Konsums und hintergründige Zwänge sowie subjektive Präferenzen berücksichtigt, damit keine wichtige Kategorie des Zielkatalogs sozialpolitischen Handelns außer Acht gelassen wird.

Entsprechende Analysen zu Armut und Ungleichheit aus einer konsumbasierten Perspektive sind in Deutschland allerdings eine Rarität. Dieser Artikel möchte die Lücke schließen und die bisher primär einkommensbasierte Armuts- und Ungleichheitsforschung durch die Konsumperspektive ergänzen und erweitern. Dabei richtet sich der Fokus der Untersuchung insbesondere auf Haushalte an den unteren Rändern der Einkommens- und Konsumverteilung. Durch die Berücksichtigung der Konsumperspektive können gegebenenfalls neue sozialpolitische Handlungsfelder identifiziert und bestehende Sozialleistungssysteme auf ihre Effektivität überprüft werden. Dafür werden die Daten der Einkommens- und Verbrauchsstichprobe (EVS) der Jahre 1978 bis 2008 auf folgende Fragen hin untersucht:

(i) Wie unterscheiden sich einkommens- und konsumbasierte Wohlstandsmessungen in der Darstellung von Armut und Ungleichheit in Deutschland?

(ii) Gibt es wohlstandsrelevante Unterschiede zwischen Einkommens- und Konsumarmen?

(iii) Kann durch die Berücksichtigung der Ausgaben bei der Armutsmessung eine Gruppe benachteiligter Haushalte identifiziert werden, die bei der alleinigen Verwendung der Einkommen unberücksichtigt bleibt?

Die Arbeit beginnt mit einem Überblick über konzeptionelle (Kapitel 2.1) und erhebungstechnische (Kapitel 2.2) Unterschiede der beiden Wohlstandsindikatoren, aus denen die forschungsleitenden Hypothesen abgeleitet wer- 
den (Kapitel 2.3). Der anschließende Abschnitt präsentiert die empirischen Befunde deutscher und internationaler Studien $\mathrm{zu}$ den Unterschieden zwischen einkommensund konsumbasierten Wohlstandsmessungen (Kapitel 3). Es folgt die Darstellung der verwendeten Daten (Kapitel 4.1), Operationalisierungen (Kapitel 4.2) und Methoden (Kapitel 4.3). Nach der Beschreibung der Ergebnisse (Kapitel 5) schließt die Arbeit mit einer Zusammenfassung und Diskussion der Ergebnisse (Kapitel 6).

\section{Konsum und Einkommen als Wohlstandsindikator}

\subsection{Konzeptionelle Unterschiede zwischen Konsum und Einkommen bei der Messung von Wohlstand}

Aus ökonomischer Perspektive steht der Begriff des Konsums ganz allgemein für den Kauf von Gütern des privaten Ge- und Verbrauchs. Die Güternachfrage wird dabei nicht nur vom Einkommen, sondern auch von den Güterpreisen, dem Bedarf des Haushalts, seinen Präferenzen, dem Vermögen, den Kreditmöglichkeiten sowie der Sparneigung beeinflusst. Erste Untersuchungen über den Zusammenhang zwischen Haushaltseinkommen und Konsumausgaben gehen zurück auf Ernst Engel, der Mitte des 19. Jahrhunderts Direktor des Königlich-Sächsischen Statistischen Bureaus war. Der deutsche Statistiker analysierte Haushaltsbücher und konstatierte auf dieser Basis bereits 1857 die Gesetzmäßigkeit, dass der Einkommensanteil, den ein Privathaushalt für die Ernährung ausgibt, mit steigendem Einkommen sinkt. Das nach ihm benannte Engel'sche Gesetz ist eines der am besten belegten empirischen Gesetze der Volkswirtschaftslehre. Wenig später formulierte Hermann Schwabe (1868) einen gesetzmäßigen Zusammenhang zwischen dem steigenden Wohlstand eines Haushalts und sinkenden Ausgabenanteilen für den Bereich des Wohnens: „Je ärmer Jemand ist, einen desto größeren Theil seines Einkommens muss er für Wohnung verausgaben.“ (Schwabe 1868: 267)

Hinsichtlich der absoluten Ausgaben betonte John Maynard Keynes (1937) ein knappes Jahrhundert nach Engel und Schwabe, dass das Einkommen eines Haushalts zwar eine entscheidende Rolle beim Konsum spiele, die Einnahmen und Ausgaben eines Haushalts allerdings nicht zwangsläufig deckungsgleich sein müssten, da Teile des Einkommens gespart werden könnten. Dementsprechend wird in der Einkommensverwendungsgleichung das
Einkommen (Y) als die Summe von Konsum (C) und Ersparnis (S) definiert: $Y=C+S$. Die Höhe der Ersparnis hängt neben der individuellen Sparneigung (z. B. aus Vorsorgeoder Vorsichtsmotiven) von der Höhe der Einkommen ab, denn jeder Haushalt muss einen Teil seiner Ausgaben für die Sicherung seines Existenzminimums ausgeben (Bögenhold \& Fachinger 2005). Je mehr Geld ihm zusätzlich zur Verfügung steht, umso variabler ist er sowohl in der Aufteilung seines Einkommens zwischen Sparen und Konsum als auch in dessen Gestaltung. Dabei ist nach Keynes davon auszugehen, dass der Anteil der Konsumausgaben am Einkommen, die sogenannte Konsumquote, mit zunehmendem Einkommen sinkt. Die Ersparnis beeinflusst wiederum die Konsummöglichkeiten eines Haushalts: Einerseits können Teile des Einkommens für den zukünftigen Konsum gespart werden, andererseits kann durch die Auflösung von Vermögen oder durch Verschuldung ein über das Einkommen hinausgehender finanzieller Rahmen für Konsumausgaben geschaffen werden. Bei der Erklärung des Zusammenhangs zwischen Konsum und Einkommen muss daher auch immer zwingend das Vermögen eines Haushalts berücksichtigt werden.

Konsum und Einkommen werden daher als zwei unterschiedliche Seiten der Medaille „soziale Ungleichheit“ betrachtet (Bögenhold \& Fachinger 2000:6). Dennoch verwenden Untersuchungen zum Thema Armut und Ungleichheit seit jeher primär das Einkommen einer Person bzw. eines Haushalts als zentralen Indikator und nehmen die Zusammenhänge von Einkommen, Konsum und Wohlstand als gegeben an. Unberücksichtigt bleibt dabei, dass durch individuelle Fähigkeiten im Umgang mit Geld und Präferenzen bei vergleichbaren Haushalten und gegebenem Einkommen vollkommen unterschiedliche Konsumprofile realisiert werden können. Über die wirkliche Stärke des Zusammenhangs von Einkommen und Konsum kann daher diskutiert werden.

Insbesondere in den USA wird seit längerem debattiert, ob die Konsumausgaben im Vergleich zum Einkommen nicht den geeigneteren Wohlstandsindikator darstellen. An der Verwendung des Einkommens als zentraler Indikator für den Lebensstandard wird dabei unter anderem bemängelt, dass dabei kurzfristige Unterschiede zwischen Personen berücksichtigt würden, die aus langfristiger Perspektive unbedeutend sein können (Brewer u.a. 2006). Temporäre Einkommensschwankungen können z. B. im Falle von Selbstständigkeit, unregelmäßigen Bonuszahlungen, kurzen Phasen der Arbeitslosigkeit oder Krankheit auftreten. Außerdem können Einkommen in Abhängigkeit von der Lebensphase variieren, wenn z. B. die Einkommenshöhe an Faktoren wie Alter, Erfahrung oder Seniorität gekoppelt ist oder die Erwerbsphase auf- 
grund von Kindererziehung, Altersteilzeit, Rente o. ä. unterbrochen oder beendet wird. Diese Einkommenseinbußen haben einen geringen Effekt auf den Lebensstandard eines Haushalts, wenn sie im Vorfeld antizipiert werden können. Diese Argumentation stützt sich auf die Annahmen der permanenten Einkommenshypothese von Friedman (1957) sowie der Lebenszyklushypothese von Modigliani und Brumberg (1954). Erstere geht davon aus, dass Haushalte ihre Konsumentscheidungen nicht auf der Basis des kurzfristig zur Verfügung stehenden Einkommens treffen, sondern sich an ihrem permanenten Einkommen orientieren. Demnach sollte das Konsumverhalten von vorübergehenden, kurzfristigen Einkommensänderungen nicht beeinflusst werden. Analog dazu argumentiert die Lebenszyklushypothese, dass Personen zukunftsorientiert handeln und mittels verstärkten Sparens und Entsparens versuchen, ihren Konsum über den Lebenszyklus hinweg zu glätten (Brzozowski \& Crossley 2011). Die Glättung erfolgt dabei zum einen aus langfristiger Perspektive über lebensphasenspezifisches Spar- und Ausgabeverhalten: Während in jungen Jahren tendenziell über den eigenen (Einkommens-)Verhältnissen gelebt wird, dienen die mittleren Jahre zum Abbau möglicher Schulden und zum Aufbau neuen Vermögens, welches wiederum im Alter zur Aufbesserung der geringen Einkommen verwendet wird. Zum anderen erfolgt sie aus der kurzfristigen Perspektive über Vermögensauflösungen oder Kreditaufnahmen, um bei hohen Einkommensschwankungen oder kurzfristiger Arbeitslosigkeit negative Auswirkungen auf den Lebensstandard abzumildern (Christoph u. a. 2014). Im Gegensatz zu dem tendenziell eher schwankenden Einkommen ist der Konsum somit weniger variabel und wird als ein besserer Indikator für das „permanente Einkommen“ und somit als besseres Maß für den Wohlstand betrachtet (Brewer u. a. 2006; Brzozowski \& Crossley 2011; Cutler \& Katz 1992; McGregor \& Barooah 1992; Meyer \& Sullivan 2003, 2011; Noll 2007; Noll \& Weick 2007; Poterba 1991; Zaidi \& de Vos 2001). In diesem Sinne wird des Weiteren angenommen, dass mit geringeren zeitlichen Schwankungen auch eine gleichmäßigere Verteilung der Verbrauchsausgaben einhergeht und Unterschiede in den Ausgaben eher Aussagen über permanente Ungleichheiten im Wohlstand liefern können als Unterschiede im Einkommen (Brewer u. a. 2006).

Als weiterer Vorteil einer Betrachtung der Konsumverwendung wird genannt, dass Konsum nicht nur zur Deckung des alltäglichen Bedarfs benötigt werde, sondern vielmehr einen Mechanismus darstelle, der sowohl soziale Beziehungen als auch soziale Kommunikation und gesellschaftliche Hierarchien organisiere (Slater 1997). Bereits Ende des 19. Jahrhunderts beschreibt Thorstein B.
Veblen (1899), amerikanischer Sozialökonom und Pionier der Konsumsoziologie, dass der Konsum im gesellschaftlichen Kontext als Mittel zur Abgrenzung (Distinktion) zu anderen sozialen Gruppen eingesetzt werden könne. In seinen Arbeiten beschäftigt er sich primär mit dem Konsumverhalten der reichen Gesellschaftsschichten, welche durch ihren aufwändigen bis verschwenderischen Konsum nach außen hin ihre gesellschaftlich herausgehobene Stellung demonstrieren. Diese Deutung von Konsum als Mittel zur Erhaltung und Etablierung von Klassenunterschieden und als Zeichen- und Bedeutungssystem findet sich auch bei Simmel (1900, 1905), Baudrillard (1988) und Bourdieu (1987). Konsum wurde in diesem Sinne lange Zeit als Mittel der Manifestation durch ökonomische Ressourcen geschaffener vertikaler und horizontaler Differenzen verstanden (Schneider 2000). In den 1970er Jahren entstanden jedoch erste Zweifel an der Prämisse der traditionellen Strukturanalysen, dass die objektiven Lebensbedingungen die subjektiven Lebensweisen prägen. Durch die Auflösung traditioneller Bindungen und eine allgemeine Steigerung des Lebensstandards nahmen die Wahlmöglichkeiten zur individuellen Lebensgestaltung zu, und es entstanden Lebensstilgruppen, die von der klassischen Sozialstrukturanalyse nicht erfasst werden konnten (Beck 1983; Hradil 1987, 1992; Zapf u. a. 1987). So wurde auch die Annahme einer stringenten Beziehung zwischen sozialer Lage - gemessen am Einkommen oder materiellen Ressourcen - und Konsumverhalten abgelöst und durch die Vorstellung einer Entschichtung des Konsums verdrängt. Der bindende Charakter von allgemeinen und rollenspezifischen Konsumnormen löse sich zunehmend auf und nehme vermehrt den Charakter von Gestaltungsnormen an (vgl. Gross 1994; Scheuch 1975). Ein Nachteil soziologischer Konsumtheorien ist allerdings, dass der Konsum nur dann als eigenständiges Phänomen verhandelt wird, wenn er neben der Deckung des Grundbedarfs auch eine soziale Funktion erfüllt (Grauel 2010). Konsumtheorien, die sich speziell mit der Bedeutung von Konsum am unteren Ende der Wohlstandsverteilung beschäftigen, liegen nicht vor.

\subsection{Erhebungsbedingte Vor- und Nachteile einer konsumbasierten Wohlstandsmessung}

Für die Verwendung des Einkommens zur Analyse von Armut und Ungleichheit spricht zwar die große Verfügbarkeit standardisierter Daten auch im internationalen Kontext, allerdings sind entsprechende Daten vor allem im unteren Einkommensbereich fehleranfällig (Brewer 
et al 2006; Meyer \& Sullivan 2003). Einkommensdaten weisen dort in der Regel hohe Nonresponse-Raten auf, da Einkommen aus der Schattenwirtschaft, kriminellen Aktivitäten oder privaten Transfers allenfalls zögerlich als Einkommen deklariert werden. Die Angabe der mittels dieser Einkommen getätigten Ausgaben ist hingegen eher unverfänglich. Selbst wenn illegale Aktivitäten wie z. B. der Kauf von Drogen nicht als Ausgaben berichtet werden, kann die Auswertung der Ausgaben in den davon nicht betroffenen Kategorien weiterhin aussagekräftige Informationen über das Wohlergehen einer Person oder eines Haushalts liefern (Meyer \& Sullivan 2003). Meyer und Sullivan (2003) weisen darauf hin, dass das Einkommen in armutsgefährdeten Bevölkerungsgruppen neben dem regulären Erwerbseinkommen häufig aus weiteren Quellen stammt, weshalb der Anteil der zu niedrig angegebenen Transfereinkommen und der nicht berichteten Einnahmen am Gesamteinkommen in dieser Gruppe besonders hoch ist. Sie beziehen sich dabei auf eine amerikanische Studie alleinerziehender Mütter im Sozialhilfebezug, nach der eine alleinerziehende Mutter maximal 9\% ihres Einkommens aus angemeldeter Erwerbstätigkeit bezieht. Der Rest bestehe zu verschiedenen Anteilen aus privater und staatlicher Unterstützung für Alleinerziehende, Lebensmittelmarken, Schwarzarbeit und Alimenten des Kindsvaters. Je weniger dieser alternativen Einkommensquellen explizit in Umfragen erhoben werden, desto unwahrscheinlicher ist eine akkurate Einkommensangabe. Die Angaben zu Steuerzahlungen werden nach Ansicht der beiden Autoren außerdem nur selten in Umfragen erhoben oder nachträglich oft fehlerhaft imputiert.

Dem wird entgegnet, dass die Erhebung von Konsumausgaben deutlich aufwendiger, kostspieliger und keineswegs fehlerfrei sei. Die Daten des Family Expenditure Survey aus Großbritannien demonstrieren beispielhaft, dass Haushalte ihre Ausgaben für Tabak und Alkohol bewusst zu niedrig angeben (Brewer et al 2006). Zu erwarten sind ebenfalls fehlende Angaben bezüglich der Ausgaben für den Kauf illegaler Güter (z. B. Drogen), die aber zumindest für die Beurteilung eines sozialpolitisch relevanten materiellen Lebensstandards von geringer Bedeutung sein sollten (Christoph u. a. 2014). Haushalte mit hohem Einkommen neigen zudem nach Ansicht von Aguior und Bils (2011) immer öfter dazu, ihre Ausgaben ganz allgemein zu niedrig anzugeben. Dieser Messfehler sollte automatisch dazu führen, dass Messungen der Konsumungleichheit zunehmend nach unten verzerrt werden.

Des Weiteren kann es auch bei den Konsumausgaben durch den Kauf von langlebigen Gebrauchsgütern zu Schwankungen kommen. Diese Güter werden zwar nur selten, dafür aber für einen relativ hohen Preis ange- schafft. Diese Überlegung veranschaulicht, dass es sich bei Konsum und Konsumausgaben streng genommen um zweierlei Dinge handelt, die aber häufig synonym verwendet werden. Für die langlebigen Gebrauchsgüter fallen die Kosten nur einmal an, der Haushalt kann jedoch von ihrem Nutzen über eine lange Zeit profitieren. Es ist somit durchaus möglich, dass ein Haushalt trotz punktuell geringer Ausgaben einen hohen Lebensstandard aufweist.

Da die Erhebung der Konsumausgaben aufwändig und kostspielig ist, liegt die Stichprobengröße meist deutlich unter der von Einkommenserhebungen (Meyer \& Sullivan 2003). Letztere erlauben somit, Strukturen mit höherer Präzision zu bestimmen, tiefer gehende Analysen von Subgruppen durchzuführen und Hypothesen mit höherer Verallgemeinerbarkeit zu testen. Meyer und Sullivan (2004) räumen allerdings ein, dass der Zugewinn an Präzision nicht so hoch ausfällt, wie der einfache Vergleich der Stichprobengröße suggeriert.

\subsection{Hypothesen zu einkommens- und konsumbasierten Ungleichheits- und Armutsmaßen}

Aufgrund der dargestellten konzeptionellen und erhebungstechnischen Unterschiede zwischen Einkommen und Konsum als Wohlstandsindikatoren wird davon ausgegangen, dass sich die auf der Basis der Konsumausgaben ermittelten Wohlstandsmaße von denen der einkommensbasierten Analyse unterscheiden. Unterschiede in den Verteilungen von Konsum und Einkommen sollten sich insbesondere aus den geringeren zeitlichen Schwankungen der Konsumausgaben, ihrer Untererfassung vor allem bei Personen im oberen Einkommensbereich und der abnehmenden marginalen Konsumneigung bei steigenden Einkommen ergeben. Bei der Armutsmessung entstehen Differenzen, da Konsumausgaben in einem gewissen Maße das Ergebnis individueller Entscheidungen sind und relativ niedrige Ausgaben somit nicht zwangsläufig mit relativ niedrigen Einkommen korrelieren müssen, zum Beispiel wenn Haushalte eine hohe Sparneigung haben oder ausreichend mit langlebigen Gebrauchsgütern ausgestattet sind. Es werden daher folgende Hypothesen aufgestellt:

Hypothese 1: Hinsichtlich der Verteilung beider Indikatoren wird davon ausgegangen, dass die Konsumausgaben weniger ungleich verteilt sind als die Einkommen.

Hypothese 1a: Vor allem diejenigen Ungleichheitsmaße, die besonders sensitiv auf Veränderungen im unteren Bereich der Verteilung reagieren, fallen auf der Basis 
der Konsumausgaben deutlich niedriger aus als auf der Basis der Einkommen.

Hypothese 2: Die auf der Basis der Konsumausgaben ermittelten Armutsquoten und Indikatoren der Armutsintensität sind niedriger als die einkommensbasierten.

Hypothese 2a: Der Unterschied zwischen einkommens- und konsumbasierten Armutsmaßen wird umso deutlicher, je sensitiver sie auf Veränderungen im unteren Bereich der Verteilung reagieren.

Hypothese 3: Die Messungen von Einkommensund Konsumarmut erfassen zum Teil unterschiedliche Personengruppen. Inwiefern sich die Gruppen Einkommens- und Konsumarmer z. B. hinsichtlich ihrer soziodemografischen Zusammensetzung, ihrer Ausstattung mit langlebigen Gebrauchsgütern oder ihrer Konsumstruktur unterscheiden, sind empirische Fragen.

\section{Forschungsstand}

Konsumausgaben sind weit mehr als das Resultat von Budgetrestriktionen, Bedarfen, Nachfrage oder Präferenzen. Vielmehr können sie als Manifestation ökonomischer und sozialer Ungleichheit sowie kultureller Unterschiede und sozialer Distinktion betrachtet werden (Noll 2007). Es ist daher überraschend, dass der Konsum in der soziologischen Forschung in Deutschland anscheinend in Vergessenheit geraten ist. Gewiss gibt es soziologische Studien, die sich in der Tradition von Simmel, Veblen oder Bourdieu mit der sozialen oder symbolischen Funktion des Konsums beschäftigen. Dem Konsum als Aspekt des Lebensstandards wurde hingegen kaum Beachtung geschenkt, so dass nur wenige empirische Studien über die Struktur und Ungleichheit von Konsumausgaben zur Verfügung stehen (Bögenhold \& Fachinger 2000; Noll \& Weick 2005a, 2005b).

Aus den einkommensbasierten Studien zur Ungleichheit und relativen Armut in Deutschland ist bekannt, dass beides in den letzten Jahrzehnten stetig zugenommen hat. Grabka und Frick (2010) sowie Grabka et al. (2012) berichten beispielsweise von einem Anstieg der relativen Einkommensarmut zwischen 1990 und 2008 von $12 \%$ auf $14 \%$. Als besonders armutsgefährdet gelten Haushalte mit Kindern, junge Erwachsene sowie Alleinerziehende. Die Ungleichheit der Einkommen verlief von Anfang der 1990er Jahre bis zum Jahr 2006 relativ konstant, stieg dann jedoch ebenfalls an (ebd.). Diese Entwicklung wurde insbesondere von einer Polarisierung der Einkommen geprägt.
Noll und Weick (2007) verglichen mit den Daten der EVS von 1983 bis 2003 die Entwicklung der Gini-Koeffizienten für die Haushaltsäquivalenzeinkommen und -ausgaben. Sie konnten zeigen, dass die Konsumausgaben in Westdeutschland weniger ungleich verteilt waren als die Einkommen. In einer früheren Arbeit (Noll \& Weick 2005b) untersuchten die beiden Autoren, ob sich die zunehmende Ungleichverteilung der Einkommen auf ihre Verwendung auswirkt. Dafür verglichen sie die Konsumstruktur verschiedener Einkommenspositionen, d.h. die Ausgabenanteile von Güterkategorien an den Gesamtausgaben. Sie kamen zu dem Ergebnis, dass untere Einkommensgruppen mit zwei Dritteln ihrer Gesamtausgaben einen hohen Anteil ihres Budgets für die Grundbedürfnisse Nahrung, Wohnen und Bekleidung ausgeben. Bei den einkommensstärkeren Haushalten betrug der Anteil hingegen weniger als die Hälfte. Aus der Betrachtung der zeitlichen Entwicklung der Konsumstrukturen verschiedener Einkommensgruppen schlussfolgerten die Autoren, dass sich ihre Ausgabenstrukturen angenähert haben, markante Unterschiede jedoch bestehen bleiben. Aus der Perspektive der Konsumstruktur findet sich somit kein Hinweis auf eine steigende Divergenz der Ausgaben. Die Befunde werden durch eine ähnliche Untersuchung von Bögenhold und Fachinger (2005) mit den Daten der Laufenden Wirtschaftsrechnung gestützt.

Noll und Weick (2005a; 2007) untersuchten für Deutschland das Phänomen des sogenannten Overspendings als Indikator für den Wohlstand eines Haushalts bzw. einer Person. Overspending tritt dann ein, wenn die Ausgaben in einer bestimmten Periode das zur Verfügung stehende Einkommen übersteigen. Im Fokus der Autoren stehen dabei insbesondere die Haushalte am unteren Ende der Einkommensverteilung. Sie konnten zeigen, dass westdeutsche Haushalte mit weniger als $60 \%$ des Medianeinkommens $108 \%$ ihrer Einkommen verkonsumierten (Noll \& Weick 2005a), bei denjenigen mit weniger als $50 \%$ der Durchschnittseinkommen waren es sogar $116 \%$ (Noll \& Weick 2007). Die zusätzlichen Ausgaben wurden größtenteils aus vorhandenen Ersparnissen finanziert (ebd.). Hinsichtlich der Frage, inwiefern Overspending als Armutshinweis interpretiert werden kann, vertreten die Autoren die Ansicht, dass „wohl kaum davon die Rede sein [könne], dass die einkommensarmen Haushalte über ihre Verhältnisse leben“ (Noll \& Weick 2005a: 5). Vielmehr stünden ihnen vielfach weniger Mittel zur Verfügung, als sie für ihre Existenzsicherung benötigten. Die Autoren stützen ihre Argumentation dabei auf das geringe Ausgabenniveau Einkommensarmer und ihren hohen Ausgabenanteil für Grundbedürfnisse. 
Im internationalen Kontext gibt es eine Vielzahl von Studien, die konsumbezogene Indikatoren zur Analyse von Niveau und Entwicklung ökonomischen Wohlstands, Ungleichheit und Armut verwenden. Für die USA liegen unter anderem durch die Arbeiten von Cutler und Katz (1992), Johnson und Shipp (1997), Johnson und Smeeding (1998), Meyer und Sullivan (2003) sowie Krueger und Perri (2006) entsprechende Forschungsergebnisse vor. Die Studien kommen fast einstimmig zu dem Ergebnis, dass die Ausgaben im Gegensatz zum Einkommen gleichmäßiger verteilt sind. Nach der Langfristanalyse von Cutler und Katz (1992) verliefen die Einkommens- und Konsumverteilungen in ihrer zeitlichen Entwicklung parallel, wobei die Konsumausgaben in den 1980er Jahren etwas ungleicher verteilt waren. Spätere Studien berichten von einer zunehmenden Differenz beider Ungleichheitsverteilungen, da die Ungleichheit der Konsumausgaben hinter der Entwicklung der Einkommensverteilung zurückblieb (Attanasio u. a. 2012; Blundell u. a. 2008; Krueger \& Perri 2006; Meyer \& Sullivan 2003). Die Armutsanalyse von Slesnick (1993) offenbart außerdem, dass durch die Verwendung von einkommensbasierten Armutsmaßen die Armut überschätzt wird und sich bei der Betrachtung von Konsumausgaben ein positiveres Armutsbild zeigt. Eine Untersuchung mit alternativen Armutsindikatoren innerhalb der Gruppe einkommens- oder konsumarmer Haushalte liefert den Autoren Meyer und Sullivan (2003) den Hinweis, dass Letztere deutlich schlechter gestellt sind. Die Autoren schlussfolgern daraus, dass der Konsum sich besser zur Messung des Wohlstands benachteiligter Haushalte eigne.

$\mathrm{Zu}$ ähnlichen Erkenntnissen gelangen auch Brzozowski und Crossley (2011) sowie Sabelhaus und Schneider (1997) auf der Basis kanadischer Daten. Sowohl die Armutsquoten als auch die Ungleichheit seien in der konsumbasierten Betrachtung niedriger. Sabelhaus und Schneider (1997: 25) verweisen daher explizit darauf, dass politische Strategien der Einkommensumverteilung und Armutsverminderung, die sich einzig auf Einkommensstatistiken stützen, nicht aussagekräftig seien: „The results indicate clearly that income is only part of the story about economic well-being - redistribution of resources should be based on a broader notion of economic well-being."

Auch im europäischen Kontext liegen Studien über die Unterschiede in der Messung von Ungleichheiten und Armut basierend auf Einkommens- und Konsumdaten vor. Beispielsweise zeigen Brewer et al. (2006) sowie Sierminska und Garner (2003) für Großbritannien, dass dort sowohl die Einkommens- als auch die Konsumungleichheit seit den 1980er Jahren steigt, die Ausgaben jedoch gleicher verteilt sind. Hinsichtlich der Armutsquoten kann jedoch von einer unterschiedlichen Entwicklung gesprochen werden. Die Entwicklung der Einkommensarmut verläuft umgekehrt u-förmig, die der Konsumarmut weist einen gemäßigten positiven linearen Trend auf. $\mathrm{Zu}$ Beginn des 21. Jahrhunderts liegt die Konsumarmut sogar über der Einkommensarmut. Als weiteren Hinweis für die unterschiedliche Armutsmessung beider Konzepte verweisen Brewer u. a. (2006) darauf, dass nur circa die Hälfte der Einkommensarmen gleichzeitig auch zu den Konsumarmen zählt. Wie Noll und Weick (2005a; 2007) für Deutschland verwenden die Autoren das Overspending zur Analyse von Armut. Im Gegensatz zu den deutschen Autoren nehmen sie allerdings davon Abstand, Overspending zwangsläufig als Hinweis auf fehlende materielle Ressourcen $\mathrm{zu}$ interpretieren, da zum einen Haushalte versuchen, ihren Konsum im Zeitverlauf zu glätten, und es zum anderen bei beiden Indikatoren zu Messfehlern kommen kann. Der Lebensstandard armer Haushalte im Overspending mag somit nicht so gering sein wie suggeriert wird. Da die Ausgaben im unteren Teil der Einkommensverteilung verhältnismäßig hoch sind, resümieren die Autoren, dass ,a more reliable picture of who is genuinely very poor may be obtained from an examination of the bottom of the spending distribution rather than the income distribution." (ebd.: 23)

\section{Daten, Operationalisierung und Methode}

\subsection{Daten und Operationalisierung}

\subsubsection{Daten: Die Einkommens- und Verbrauchsstichprobe}

Die Einkommens- und Verbrauchsstichprobe (EVS) ist eine der wenigen amtlichen Statistiken in Deutschland, die sowohl die Einnahmen als auch die Ausgaben eines Haushalts detailliert erfasst. Darüber hinaus enthält sie Informationen über die Schuldensituation und die Ausstattung der Haushalte mit langlebigen Gebrauchsgütern. Die EVS ist eine Quotenstichprobe und wird alle fünf Jahre erhoben. Für die Analysen werden die Daten der Jahre 1978 bis 2008 verwendet, wobei jedes Jahr aus einem Querschnitt mit bis zu 60.000 Haushalten besteht. Die Konstruktion eines Haushaltspanels ist daher nicht möglich. Bei Längsschnittbetrachtungen muss somit beachtet werden, dass zwischen den Zeitpunkten variierende Befunde durch die unterschiedliche Stichprobenzusammensetzung bedingt sein können. Da es sich bei der EVS allerdings um repräsentative Stichproben handelt, sollten die konstatierten 
Entwicklungen nur marginal auf Stichprobenfehler zurückzuführen sein.

Die EVS begrenzt ihre Stichproben auf Haushalte mit einem bestimmten Einkommen, weshalb Bezieher extrem hoher Einkommen unterrepräsentiert sind und der EVS im Allgemeinen ein Mittelstandsbias und mangelnde Repräsentativität an den Rändern der Einkommensverteilung nachgesagt wird, deren Auswirkungen jedoch nicht quantifizierbar sind (Becker \& Schüssler 2014).

Innerhalb des Beobachtungszeitraums kam es mehrfach zu Veränderungen sowohl bei der Zusammensetzung der Stichprobe als auch bei der Erhebung und Klassifikation der Einkommen und Konsumausgaben. Die Zusammensetzung der Stichprobe änderte sich ab 1993, als erstmals ostdeutsche Befragte teilnahmen. Zeitgleich wurde die Stichprobe um Haushalte mit ausländischem Haushaltsvorstand erweitert. Die Teilpopulation der ausländischen Bezugspersonen wird dabei nicht gesondert hochgerechnet, wodurch Schätzungen zu Ungleichheit, Armut und Reichtum auf der Basis der EVS tendenziell zu gering ausfallen (Becker \& Hauser 2004). Für die folgenden Analysen wurde die Stichprobe daher auf deutsche Haushalte aus Westdeutschland beschränkt. Generell ist bei derart aufwendigen Erhebungen davon auszugehen, dass es zu einer gewissen Selektivität der Stichprobe kommt (ebd.).

1998 erfolgte eine Umstellung von Jahres- zu Quartalswerten bei der Erhebung der Einnahmen und Ausgaben, so dass seitdem unregelmäßig anfallende Einkommensund Ausgabearten nur unzureichend erfasst werden. Die Einnahmen und Ausgaben der verschiedenen Quartalspopulationen sind zudem aufgrund saisonaler Schwankungen und unregelmäßig anfallender Bezüge nur begrenzt vergleichbar (ebd.). Becker und Hauser (2004) zeigen jedoch, dass sich quartalsspezifische und -unabhängige Berechnungen nur unwesentlich unterscheiden.

Bei den Konsumausgaben, die in der EVS anhand eines tiefgegliederten Klassifikationssystems erhoben werden, wurde das Frageprogramm mehrfach an aktuelle Gegebenheiten und Entwicklungen angepasst. Neue Konsumtrends ersetzten Güter, deren Verbreitungsgrade über die Haushalte stagnierten, und erweiterten die Liste der Ausstattungsgegenstände. Das Systematische Verzeichnis der Einnahmen und Ausgaben der privaten Haushalte (SEA) unterteilt die Konsumausgaben nach Verwendungszwecken und wurde zum Jahr 1998 an das internationale Klassifikationssystem der COICOP (Classification of Individual Consumption by Purpose) angepasst. Zur Vergleichbarkeit mit früheren Erhebungen wurden die Klassifikationsschemata vor 1998 soweit möglich an das SEA98 angepasst.

\subsubsection{Operationalisierung: Konsum und Einkommen}

\section{Konsum}

Die Angaben zu den Konsumausgaben in der EVS beruhen auf dem Marktentnahmekonzept, d.h. es werden nur Käufe und Ausgaben verzeichnet, die am Markt realisiert wurden. Berücksichtigt werden dabei auch unterstellte Werte wie z.B. der Mietwert von Eigentumswohnungen. Nicht einberechnet werden hingegen Zahlungen von direkten Steuern, Versicherungen, Übertragungen an andere private Haushalte oder Organisationen, Verzinsungen und Tilgungen von Krediten, Käufe von Grundstücken und Gebäuden sowie Ausgaben für die Bildung von Geldvermögen. Vom Staat kostenlos oder vergünstigt zur Verfügung gestellte Leistungen wie allgemeine Schulbildung oder Gesundheitsdienstleistungen werden ebenfalls nicht berücksichtigt.

In Analysen zur Konsumstruktur werden folgende elf Konsumbereiche unterschieden: Ausgaben für Nahrungsmittel, Tabakwaren und (alkoholische) Getränke (NGT), Ausgaben für Bekleidung und Schuhe (Kleidung), Ausgaben für Wohnen und Energie (Wohnen), Ausgaben für Einrichtungsgegenstände, Geräte und Ausrüstungen für den Haushalt sowie deren Instandhaltung (Innenausstattung), Ausgaben für die Gesundheitspflege (Gesundheit), Ausgaben für Verkehr (Verkehr), Ausgaben für Nachrichtenübermittlung (Kommunikation), Ausgaben für Freizeit, Kultur und Unterhaltung (Freizeit), Ausgaben für Bildung (Bildung), Ausgaben für Beherbergungs- und Gaststättendienstleistungen (Gast) sowie Ausgaben für andere Waren und Dienstleistungen (Sonstiges).

\section{Einkommen}

Für die Berechnung von einkommensbasierten Ungleichheits- und Armutsmaßen werden die verfügbaren bzw. ausgabefähigen Einkommen und Einnahmen verwendet. Diese setzen sich aus dem Haushaltsnettoeinkommen und den Einnahmen aus dem Verkauf von Waren sowie den sonstigen Einnahmen zusammen. Nicht berücksichtigt werden Einnahmen aus der Auflösung und Umwandlung von Vermögen sowie der Kreditaufnahme. Haushalten in selbstgenutztem Wohneigentum wird eine fiktive Miete (auch Eigentümermietwert) unterstellt.

Um die Ausgaben und Einnahmen von Haushalten mit unterschiedlicher Größe und Struktur vergleichbar zu machen, werden diese mit Hilfe der neuen OECD-Äquivalenzskala bedarfsgewichtet (vgl. Hagenaars u. a. 1994). Für alle Analysen wird der am Mikrozensus orientierte Hochrechnungsfaktor verwendet, welcher statistische Aussagen auf der Ebene von Personen erlaubt (vgl. Noll \& Weick 2007). 


\subsection{Methode}

\subsubsection{Ungleichheitsmaße}

In der Verteilungstheorie wurde eine Vielzahl von Maßzahlen mit unterschiedlichen Eigenschaften entwickelt, die ganz allgemein den Grad der Ungleichverteilung einer bestimmten Größe in der Grundgesamtheit beschreiben. Aussagekräftig ist dabei weniger das jeweilige absolute Niveau der Indikatoren als ihre Veränderungen im Zeitverlauf oder Abstände zu einem Vergleichswert auf einer anderen Verteilungsebene (Becker \& Hauser 2004). Jedem Messkonzept liegt eine normative Annahme in der Form der unterstellten sozialen Wohlfahrtsfunktion zugrunde, d. h. sie reagieren unterschiedlich empfindlich auf Verteilungsänderungen in bestimmten Bereichen der Verteilung (ebd.). Um verschiedene Wertvorstellungen abzudecken, werden mehrere Ungleichheitsmaße berechnet und ausgewiesen.

Zum einen werden mehrere Perzentilvergleiche vorgenommen, eine sehr einfache Möglichkeit der Analyse der Verteilung einer Ressource darstellen. Die Ressourcenausstattung zweier Perzentile wird dabei in Relation gesetzt und ein niedriger Quotient als Annäherung beider Perzentile und zunehmende Gleichverteilung der Ressourcen interpretiert.

Zum anderen werden die Perzentilvergleiche durch komplexere statistische Maße der Ungleichverteilung ergänzt. Neben dem bekannten Gini-Koeffizienten, der Werte zwischen 0 (vollkommene Gleichverteilung) und 1 (vollkommene Ungleichverteilung) annehmen kann, werden mit dem Theil-Index und dem Atkinson-Maß zwei weitere Ungleichheitsmaße berechnet, die sensibler als der GiniKoeffizient auf Änderungen an den Rändern der Verteilung reagieren (Becker \& Hauser 1994; Cowell \& VictoriaFeser 1996).

Der Theil-Index beschreibt das Verhältnis von Ressourcen- und Populationsanteilen und kann bei $n$ Beobachtungen Werte zwischen 0 und $\ln (n)$ annehmen. Ist das Verhältnis ausgeglichen, herrscht keine Ungleichheit und der Index liegt bei 0. Besitzt jedoch ein kleiner Teil der Bevölkerung einen Großteil der Ressourcen, liegt Ungleichheit vor und der Index nähert sich $\ln (n)$ an (Anand 2001). Der Theil-Index ist sensitiv gegenüber Veränderungen insbesondere am oberen Rand der Verteilung und anfällig für Ausreißer. Das Atkinson-Maß beruht auf einer sozialen Wohlfahrtsfunktion, bei welcher anhand des sogenannten Ungleichheitsaversionsparameters $\varepsilon$ berücksichtigt werden kann, inwieweit die Gesellschaft Ungleichheit ablehnt. Je nachdem wie $\varepsilon$ gewählt wird, ist das AtkinsonMaß eher sensitiv gegenüber Veränderungen im unteren, mittleren oder oberen Bereich der Verteilung (Becker \& Hauser 1994; Lüthi 1981). Je höher $\varepsilon$ gewählt wird, desto stärker ist die angenommene Ungleichheitsaversion, umso stärker fallen Diskrepanzen im unteren Bereich der Verteilung gegenüber dem mittleren Bereich ins Gewicht und umso stärker steigt der Indikatorwert bei zunehmender Benachteiligung der untersten Schichten (Becker \& Hauser 1994). Der Wertebereich liegt unabhängig vom gewählten $\varepsilon$ zwischen 0 (vollkommene Gleichverteilung) und 1 (vollkommene Ungleichverteilung).

\subsubsection{Armutsmaße}

Die Definition von Armut ist ohne normative Setzungen nicht möglich, und bei der Herleitung und Konkretisierung eines jeden Armutskonzepts fließen diese mit ein (Becker \& Hauser 2004). Wie zuvor bei den Ungleichheitsmaßen ist es auch hier angebracht, über verschiedene Armutsindikatoren eine größere Bandbreite an normativen Vorstellungen $\mathrm{zu}$ berücksichtigen. Es kann somit untersucht werden, ob die beobachtete Armutsentwicklung von der normativen Setzung abhängt oder aber einem einheitlichen Trend folgt (ebd.). Als Armutsindikatoren werden die FGT-Maße sowie der Sen-Index verwendet.

$\mathrm{Zu}$ den FGT-Maßen zählen neben der geläufigen Armutsquote auch Kennziffern der Armutsintensität (vgl. Foster u. a. 1984). Die Armutsquote (head count ratio; FGT(0)) gibt den Anteil der Bevölkerung an, der unter einer festgelegten Armutsgrenze liegt, und gilt als ein sehr einfaches Maß für die Betroffenheit von Armut. Sie misst dabei allerdings nicht den Abstand der Personen unterhalb der Grenze zu dieser, also die Intensität der Armut. Werden die Menschen unterhalb der Grenze ärmer, dann spiegelt sich das nicht in der Armutsquote wieder. Als Armutsgrenze wird häufig der Geldbetrag festgelegt, der bei $60 \%$ des Medians der bedarfsgewichteten Einkommen liegt. Die folgenden Armutsanalysen beziehen sich ebenfalls auf die Armutsgrenze von weniger als $60 \%$ der bedarfsgewichteten, verfügbaren Einkommen (Median) bzw. der bedarfsgewichteten Konsumausgaben (Median).

Die relative Armutslücke (FGT(1)) gibt Aufschluss über die Intensität der Armut, indem sie den durchschnittlichen Abstand der Einkommen der als arm definierten Personen zu der Armutsgrenze misst und diesen in Relation zur Armutsgrenze setzt. Um auch die Ungleichheit innerhalb der Armutspopulation zu berücksichtigen, werden bei dem zweiten FGT-Maß zur Armutsintensität (FGT(2)) die prozentualen Abstände zur Armutsschwelle quadriert. Dadurch werden diejenigen innerhalb der Armutspopula- 
Tab. 1: Ungleichheitsmaße zur Verteilung der Einkommen und Konsumausgaben von 1978 bis 2008

\begin{tabular}{lrrrrrrr}
\hline & $\mathbf{1 9 7 8}$ & $\mathbf{1 9 8 3}$ & $\mathbf{1 9 8 8}$ & $\mathbf{1 9 9 3}$ & $\mathbf{1 9 9 8}$ & $\mathbf{2 0 0 3}$ & $\mathbf{2 0 0 8}$ \\
\hline Einkommen & & & & & & & \\
p90/p10 & 2,90 & 2,95 & 3,04 & 3,40 & 3,24 & 3,36 & 3,50 \\
p90/p50 & 1,77 & 1,75 & 1,79 & 1,86 & 1,85 & 1,85 & 1,78 \\
p10/p50 & 0,61 & 0,59 & 0,59 & 0,55 & 0,57 & 0,55 & 0,51 \\
Gini & 0,247 & 0,243 & 0,253 & 0,269 & 0,265 & 0,270 & 0,266 \\
Theil-Index & 0,106 & 0,101 & 0,109 & 0,121 & 0,119 & 0,125 & 0,119 \\
A(0.5) & 0,050 & 0,048 & 0,052 & 0,058 & 0,057 & 0,059 & 0,058 \\
A(1) & 0,094 & 0,092 & 0,099 & 0,112 & 0,109 & 0,114 & 0,114 \\
A(2) & 0,173 & 0,172 & 0,183 & 0,216 & 0,206 & 0,220 & 0,230 \\
\hline Konsum & & & & & & & \\
p90/p10 & 2,95 & 2,77 & 2,86 & 2,93 & 2,98 & 2,97 & 2,93 \\
p90/p50 & 1,75 & 1,69 & 1,74 & 1,76 & 1,83 & 1,82 & 1,73 \\
p10/50 & 0,60 & 0,61 & 0,61 & 0,60 & 0,61 & 0,61 & 0,59 \\
Gini & 0,240 & 0,226 & 0,236 & 0,240 & 0,266 & 0,264 & 0,252 \\
Theil-Index & 0,095 & 0,084 & 0,093 & 0,095 & 0,130 & 0,131 & 0,115 \\
A(0.5) & 0,046 & 0,041 & 0,045 & 0,046 & 0,059 & 0,059 & 0,053 \\
A(1) & 0,089 & 0,079 & 0,086 & 0,088 & 0,109 & 0,109 & 0,099 \\
A(2) & 0,167 & 0,151 & 0,161 & 0,165 & 0,190 & 0,189 & 0,179 \\
\hline
\end{tabular}

Quelle: EVS-Daten 1978-2008, eigene Berechnungen.

tion stärker gewichtet, die intensiv von Armut betroffen sind.

Ergänzt werden die FGT-Maße durch den Sen-Index, einen multidimensionalen Armutsindex (vgl. Sen 1976). Er basiert auf einer Kombination aus Armutsquote, Armutslücke und Gini-Koeffizient und kann als eine mit dem Gini-Index gewichtete Summe der Armutsquote und der Armutslücke verstanden werden. Somit werden sowohl das Ausmaß der Armut als auch die Ungleichheit berücksichtigt. Der Sen-Index wird außerhalb des akademischen Kontextes selten verwendet, da er im Vergleich zu anderen Armutsmaßen weniger intuitiv verständlich ist (Haughton \& Khandker 2009).

Die genannten Indikatoren können nur einen Aspekt von Armut im Sinne des Lebenslagenkonzepts abdecken. Ergänzend werden daher auch andere Unterversorgungslagen wie z. B. Verschuldung, schlechte Wohnsituation sowie Defizite in der Ausstattung des Haushalts betrachtet.

\section{Ergebnisse}

\subsection{Die Entwicklung der Ungleichheit aus der Einkommens- und Konsumperspektive}

Tabelle 1 liefert einen Überblick über die zeitliche Entwicklung verschiedener Ungleichheitsmaße und differenziert dabei nach der Verteilung von Einkommen und Kon- sumausgaben in Deutschland. Bis zum Jahr 2003 lässt sich ein deutlicher Trend zu steigender Ungleichheit der Einkommen feststellen. Im weiteren Verlauf stagniert diese Entwicklung oder ist rückläufig, bleibt allerdings zumeist über dem Niveau von 1978. Die Perzentilverhältnisse zeigen dabei eine steigende Spreizung der Einkommen, d.h. der Abstand der Einkommen der oberen 90 \% der Einkommensverteilung zu den unteren $10 \%$ vergrößert sich stetig. Im Jahr 2008 verdienten die oberen $90 \%$ das 3,5fache der unteren $10 \%$, 30 Jahre zuvor war es nur das 2,9fache. Ursache für die steigende Differenz ist ein Rückgang der Einkommen der Personen am unteren Ende der Einkommensverteilung, da sich das Einkommen der oberen $90 \%$ im Vergleich zum Medianeinkommen nur marginal verändert hat. Die weiteren Ungleichheitsmaße zeigen einen ähnlichen Verlauf an. Eine Ausnahme stellt dabei das Atkinson-Maß dar, welches besonders sensitiv auf Veränderungen im unteren Bereich der Einkommensverteilung reagiert. Die zunehmende Ungleichverteilung der Einkommen zu Ungunsten der untersten Einkommensschichten führt dazu, dass dieser Index auch zwischen den Jahren 2003 und 2008 zugenommen hat.

Die Entwicklung der Ungleichverteilung der Konsumausgaben unterscheidet sich von der der Einkommen. Zum einen sind die Konsumausgaben insgesamt weniger ungleich verteilt als die Einkommen, zum anderen zeichnet sich die Konsumungleichheit durch einen deutlich unsteteren zeitlichen Verlauf aus. Die Spreizung der Ausgaben ist im Vergleich zum Einkommen zwischen 1978 und 
Tab. 2: Relative Konsumposition in Abhängigkeit von der relativen Einkommensposition (jeweils in Prozent des Medians), 1978 und 2008 , Angaben in Prozent

\begin{tabular}{|c|c|c|c|c|c|c|c|c|c|c|c|c|}
\hline \multirow[b]{3}{*}{ Konsumposition } & \multicolumn{12}{|c|}{ Einkommensposition } \\
\hline & \multirow[b]{2}{*}{1978} & \multirow{2}{*}{$\begin{array}{r}<50 \% \\
2008\end{array}$} & \multicolumn{2}{|c|}{$50-70 \%$} & \multicolumn{2}{|c|}{$70-110 \%$} & \multicolumn{2}{|c|}{$110-150 \%$} & \multicolumn{2}{|c|}{$150-200 \%$} & \multirow{2}{*}{\multicolumn{2}{|c|}{$\begin{array}{r}>200 \% \\
2008\end{array}$}} \\
\hline & & & 1978 & 2008 & 1978 & 2008 & 1978 & 2008 & 1978 & 2008 & & \\
\hline$<50 \%$ & 46 & 38 & 12 & 5 & 2 & 1 & $<1$ & $<1$ & $<1$ & $<1$ & $<1$ & $<1$ \\
\hline $50-70 \%$ & 40 & 44 & 43 & 37 & 15 & 11 & 3 & 3 & 1 & 1 & $<1$ & 1 \\
\hline $70-110 \%$ & 13 & 15 & 41 & 50 & 58 & 60 & 33 & 37 & 14 & 19 & 6 & 11 \\
\hline $110-150 \%$ & 1 & 2 & 3 & 6 & 21 & 22 & 41 & 39 & 33 & 39 & 18 & 24 \\
\hline $150-200 \%$ & $<1$ & 1 & $<1$ & 2 & 3 & 4 & 18 & 15 & 34 & 27 & 32 & 28 \\
\hline$>200 \%$ & $<1$ & $<1$ & $<1$ & 1 & 1 & 2 & 4 & 6 & 17 & 14 & 43 & 35 \\
\hline
\end{tabular}

Quelle: EVS 1978 und 2008, eigene Berechnungen.

2008 sogar marginal rückläufig: Zu beiden Zeitpunkten geben die oberen $90 \%$ der Konsumverteilung knapp das Dreifache der unteren $10 \%$ aus. Auch die Relationen des Median zu dem 90 \%- bzw. $10 \%$-Perzentil bleiben nahezu konstant. Die Atkinson-Indizes deuten an, dass die Ungleichheit über die Zeit hinweg zwar zugenommen hat, der untere Teil der Konsumverteilung aber nicht besonders stark betroffen ist.

Die Hypothese einer weniger ungleichen Verteilung der Konsumausgaben kann somit bestätigt werden (Hypothese 1). Die Differenz wird umso deutlicher, je sensitiver das Ungleichheitsmaß auf Veränderungen im unteren Bereich der Verteilung reagiert (Hypothese 1a).

Eine Gegenüberstellung der relativen Einkommensund Ausgabenpositionen gibt weitere Hinweise auf Unterschiede zwischen beiden Verteilungen (vgl. Tab.2). Aus Platzgründen wird sich auf das erste und letzte Beobachtungsjahr beschränkt. Die Ergebnisse verdeutlichen, dass nur eine Minderheit der Personen, die sich in den unteren Einkommenspositionen befinden, auch $\mathrm{zu}$ den unteren Ausgabenpositionen zählt. Von den Personen mit weniger als 50\% des Medianeinkommens gehörten 1978 nur knapp die Hälfte auch zu der Gruppe mit weniger als $50 \%$ der Medianausgaben, im Jahr 2008 sind es sogar weniger als $40 \%$. Auch bei den Personen mit 50-70\% des Medianeinkommens zeigt sich ein ähnliches Bild. Bei der ausgabenbasierten Betrachtung steht somit in diesen Segmenten der Verteilung mehr als die Hälfte der Personen relativ gesehen besser da als bei der einkommensbasierten Betrachtung.

Die Mehrzahl der Personen im höheren Einkommensbereich gehört allerdings in der Regel zu einer niedrigeren Konsumposition. 1978 zählte noch die Hälfte der Personen mit einem Einkommen von 150-200\% des Medianeinkommens zu den Konsumgruppen mit mehr als $150 \%$ der
Medianausgaben, 2008 waren es $40 \%$. In der Gruppe mit den höchsten Einkommen können nur knapp 40 \% (1978) bzw. $35 \%$ (2008) der höchsten relativen Konsumposition zugeordnet werden. Dies entspricht allerdings der durch Keynes bekannten Gesetzmäßigkeit, dass der Anteil der Konsumausgaben am Einkommen mit steigendem Einkommen sinkt.

\subsection{Entwicklung der Armut aus der Ein- kommens- und der Konsumperspektive}

Die Ergebnisse der Armutsanalyse hinsichtlich Niveau und Entwicklung variieren ebenfalls deutlich zwischen den beiden Indikatoren. Abbildung 1 demonstriert anhand dreier Mengendiagramme für die Jahre 1978, 1993 und 2008, inwieweit sich die anhand beider Indikatoren ermittelten Armutspopulationen entsprechen. Die Größe der Kreise und die angegebenen Prozentpunkte beziehen sich jeweils auf den Anteil Einkommens- bzw. Konsumarmer an der Gesamtbevölkerung. Der Bereich, in dem sich beide Kreise überlappen, steht für diejenigen Personen, die im Sinne beider Armutsmaße als arm bezeichnet werden können.

Unabhängig vom verwendeten Indikator ist eine $\mathrm{Zu}$ nahme der Armut in Deutschland zu verzeichnen. Die auf der Basis der Einkommen berechneten Armutsquoten indizieren dabei fast durchgängig ein höheres Armutsniveau. Nur 1978 lag der Anteil Konsumarmer über dem Anteil Einkommensarmer, danach stieg der Anteil Einkommensarmer deutlich stärker an als der der Konsumarmen, so dass die Differenz beider Armutsquoten 2008 ca. fünf Prozentpunkte betrug. Wer konsumarm ist, ist demnach nicht zwangsläufig auch einkommensarm und umgekehrt (Hypothese 3). Der Prozentsatz sowohl einkommens- als 
Anteil Einkommens- und Konsumarme 1978

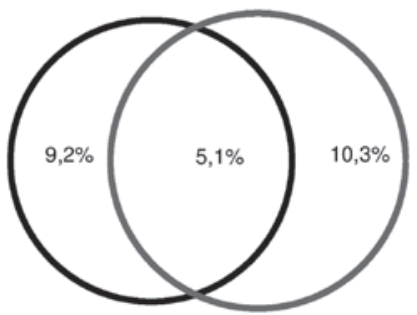

einkommensarm

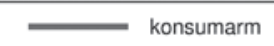

Anteil Einkommens- und Konsumarme 1993

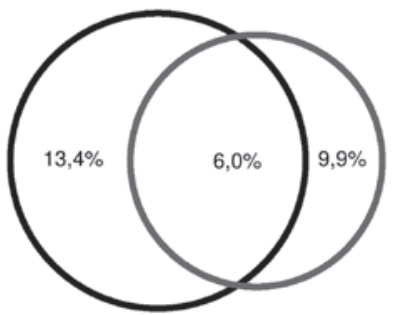

einkommensarm

konsumarm

Anteil Einkommens- und Konsumarmer 2008

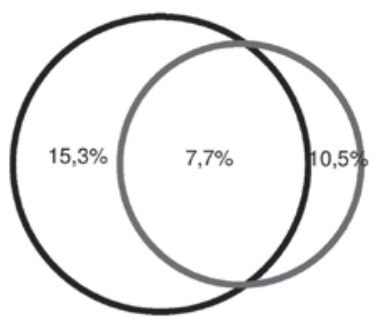

einkommensarm

konsumarm

Abb. 1: Anteil Einkommens- und Konsumarmer in den Jahren 1978, 1993 und 2008, Quelle: EVS 1978, 1993, 2008; eigene Berechnungen.

Tab. 3: Armutsquoten ( $<60 \%$ Median) basierend auf Einkommen und Verbrauch von 1978-2008 (in \%)

\begin{tabular}{llrrrrrrr}
\hline & & $\mathbf{1 9 7 8}$ & $\mathbf{1 9 8 3}$ & $\mathbf{1 9 8 8}$ & $\mathbf{1 9 9 3}$ & $\mathbf{1 9 9 8}$ & $\mathbf{2 0 0 3}$ & $\mathbf{2 0 0 8}$ \\
\hline FGT(0) & Einkommen & 9,2 & 10,5 & 10,7 & 13,4 & 12,2 & 13,2 & 15,3 \\
& Konsum & 10,3 & 9,4 & 9,4 & 9,9 & 9,2 & 9,1 & 10,5 \\
\hline \multirow{2}{*}{ GGT(1) } & Einkommen & 16,0 & 17,7 & 18,4 & 19,6 & 20,5 & 22,1 & 25,1 \\
& Konsum & 17,0 & 16,6 & 16,7 & 15,8 & 16,2 & 16,4 & 17,4 \\
\hline \multirow{2}{*}{ GT(2) } & Einkommen & 4,2 & 5,1 & 5,4 & 6,0 & 6,8 & 7,6 & 9,4 \\
& Konsum & 4,6 & 4,5 & 4,5 & 4,0 & 4,3 & 4,3 & 4,7 \\
\hline \multirow{2}{*}{ Sen-Index } & Einkommen & 0,154 & 0,170 & 0,177 & 0,189 & 0,195 & 0,210 & 0,238 \\
& Konsum & 0,164 & 0,160 & 0,161 & 0,153 & 0,157 & 0,158 & 0,168 \\
& & & & & & & &
\end{tabular}

Quelle: EVS 1978-2008, eigene Berechnungen

auch konsumarmer Personen stieg allerdings zwischen 1978 und 2008 von 5\% auf fast $8 \%$ der Gesamtbevölkerung bzw. von $35 \%$ auf $43 \%$ unter allen Armen. Im Zeitverlauf kommt es somit zu einer Angleichung beider Armutspopulationen.

Die bis hierher betrachteten Armutsquoten berücksichtigen jedoch nicht die Verteilung der Personen unterhalb der Armutsschwelle und ihren Abstand zur Armutsgrenze. Zusätzlich werden daher noch die relativen Armutslücken (FGT(1) und FGT(2)) sowie der Sen-Index ausgewiesen, die diese Punkte explizit modellieren (vgl. Tab.3). Die einfache relative Armutslücke (FGT(1)) auf
Einkommensbasis weist dabei einen linearen Anstieg um neun Prozentpunkte zwischen 1978 und 2008 auf. Das Pendant auf der Basis der Konsumausgaben beginnt 1978 auf einem leicht höheren Niveau, verläuft im Folgenden allerdings u-förmig und erreicht 2008 ungefähr das Ausgangsmaß. Mit Ausnahme von 1978 liegt die konsumbasierte Armutslücke immer - und zum Teil deutlich - unter der einkommensbasierten, d. h. die Armutsintensität ist gemessen am Konsum niedriger. Für das FGT(2)-Maß und den Sen-Index, welche besonders sensitiv auf Änderungen der Einkommen am unteren Ende der Verteilung reagieren, ist die Differenz zwischen beiden Indikatoren 
Tab. 4: Armutsrisiken soziodemografischer Gruppen nach Wohlstandsindikator, Logistische Regression, Odds Ratios, Standardfehler (geclustert) in Klammern

\begin{tabular}{|c|c|c|c|c|c|c|}
\hline \multirow[b]{2}{*}{ Konstante } & \multicolumn{2}{|c|}{ einkommensarm } & \multicolumn{2}{|c|}{ konsumarm } & \multicolumn{2}{|c|}{ nur-konsumarm } \\
\hline & $0,19 * \star \star$ & $(0,04)$ & $0,14^{\star \star \star}$ & $(0,02)$ & $0,05^{\star \star \star}$ & $(0,00)$ \\
\hline \multicolumn{7}{|l|}{ Alter (Referenz: <30 Jahre) } \\
\hline 30-39 Jahre & $0,47^{\star \star \star}$ & $(0,02)$ & $0,55^{\star \star \star}$ & $(0,01)$ & $0.64^{\star \star \star}$ & $(0,02)$ \\
\hline 40-49 Jahre & $0,36^{\star \star \star}$ & $(0,04)$ & 0,46 *** & $(0,04)$ & $0,54^{\star \star \star}$ & $(0,05)$ \\
\hline 50-59 Jahre & $0,28^{\star \star \star}$ & $(0,04)$ & 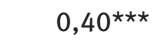 & $(0,05)$ & $0,55^{\star \star \star}$ & $(0,05)$ \\
\hline 60-69 Jahre & $0,21^{\star \star \star}$ & $(0,02)$ & $0,32^{\star \star \star}$ & $(0,04)$ & $0,62^{\star \star \star}$ & $(0,09)$ \\
\hline$\geq 70$ Jahre & $0,27^{\star \star \star}$ & $(0,04)$ & $0,55^{\star \star}$ & $(0,11)$ & 1,30 & $(0,23)$ \\
\hline \multicolumn{7}{|c|}{ Haushaltstyp (Referenz: Single-Haushalt) } \\
\hline Paar-Haushalt & $0,46^{\star \star \star}$ & $(0,02)$ & $0,48^{\star \star \star}$ & $(0,02)$ & $0,71^{\star \star \star}$ & $(0,03)$ \\
\hline Familie + 1 Kind & $0,54^{\star \star \star}$ & $(0,04)$ & $0,66^{\star * *}$ & $(0,04)$ & 1,09 & $(0,06)$ \\
\hline Familie + 2 Kinder & 0,89 & $(0,09)$ & 1,09 & $(0,09)$ & $1,55^{\star \star \star}$ & $(0,08)$ \\
\hline Familie $+3^{+}$Kinder & $1,61^{\star \star \star}$ & $(0,21)$ & $2,10^{\star \star \star}$ & $(0,23)$ & $2,65^{\star \star \star}$ & $(0,21)$ \\
\hline Alleinerziehend + 1 Kind & $1,65^{\star \star \star}$ & $(0,19)$ & 1,19 & $(0,11)$ & 1,06 & $(0,13)$ \\
\hline Alleinerziehend + 2 Kind & $2,43^{\star * \star}$ & $(0,21)$ & $1,71^{\star * *}$ & $(0,17)$ & 1,50 ** & $(0,22)$ \\
\hline Alleinerziehend $+3^{+}$Kind & $2,74^{\star \star \star}$ & $(0,39)$ & $2,37^{\star \star \star}$ & $(0,33)$ & $2,13^{\star \star \star}$ & $(0,43)$ \\
\hline Sonstige & 1,08 & $(0,19)$ & $1,63^{\star \star \star}$ & $(0,22)$ & $2,31^{\star \star \star}$ & $(0,24)$ \\
\hline \multicolumn{7}{|c|}{ Erwerbsstatus (Referenz: erwerbstätig) } \\
\hline Arbeitslos & 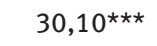 & $(4,49)$ & $14,70 * \star \star$ & $(2,34)$ & 0,86 & $(0,17)$ \\
\hline Rentner/Pensionär & $5,99 * \star \star$ & $(0,50)$ & $3,42^{\star \star \star}$ & $(0,22)$ & 1,17 & $(0,19)$ \\
\hline Geschlecht (Referenz: Mann) & $1,38^{\star \star \star}$ & $(0,05)$ & 1,06 & $(0,02)$ & $0,85^{\star \star \star}$ & $(0,03)$ \\
\hline $\mathrm{N}$ & 247855 & & 247855 & & 247855 & \\
\hline Pseudo $\mathrm{R}^{2}$ & 0,14 & & 0,09 & & 0,02 & \\
\hline
\end{tabular}

${ }^{* \star \star} p<0,001 ;{ }^{* \star} p<0,01 ;{ }^{*} p<0,05$, Quelle: EVS 1978-2008; eigene Berechnungen.

noch ausgeprägter. Die Annahmen einer niedrigeren Armutsquote und -intensität bei der konsumbasierten Armutsanalyse allgemein (Hypothese 2) und insbesondere bei Berücksichtigung der Ungleichheit der Armen (Hypothese $2 a$ ) werden somit bestätigt.

\subsection{Merkmale Einkommens- und Konsumarmer im Vergleich}

Wie gezeigt, überschneiden sich die Personenkreise Einkommens- und Konsumarmer nur partiell. Ein Teil der Konsumarmen würde somit bei einer rein einkommensbasierten Armutsanalyse unberücksichtigt bleiben. Sollten diese Personen zumindest ähnliche Benachteiligungen wie die Einkommensarmen aufweisen, kann dies als Indiz dafür betrachtet werden, dass die reine Betrachtung der Einkommen bei der Bestimmung von Armut unzureichend ist. Für die folgenden Analysen werden daher drei verschiedene Armutspopulationen unterschieden: (1) Einkommensarme, (2) Konsumarme sowie (3) diejenigen Konsumarmen, die nicht zugleich zu den Einkommensar- men zählen. Letztere werden auch als Nur-Konsumarme bezeichnet.

\section{Armutsrisiken soziodemografischer Gruppen nach Wohlstandsindikator}

Tabelle 4 gibt die Ergebnisse dreier logistischer Regressionen hinsichtlich der Armutsrisiken verschiedener soziodemografischer Gruppen in Abhängigkeit vom verwendeten Wohlstandsindikator an. Unabhängig vom gewählten Indikator haben junge Personen unter 30 Jahren sowie Alleinerziehende und Familien mit mehreren Kindern ein höheres Armutsrisiko. Während das Risiko der Einkommensarmut mit dem Alter stetig sinkt, zeigt sich bei der Konsumarmut ein leicht u-förmiger Verlauf. Bei den NurKonsumarmen tritt der u-förmige Verlauf deutlicher zum Vorschein, denn die Armutsrisiken der jüngsten und ältesten Haushalte unterscheiden sich nicht mehr signifikant.

Deutliche Unterschiede zwischen den Armutsrisiken sieht man zum einen beim Erwerbsstatus und zum anderen beim Geschlecht des Haushaltsvorstands. Während bei der Einkommens- und Konsumarmut das Armutsrisi- 


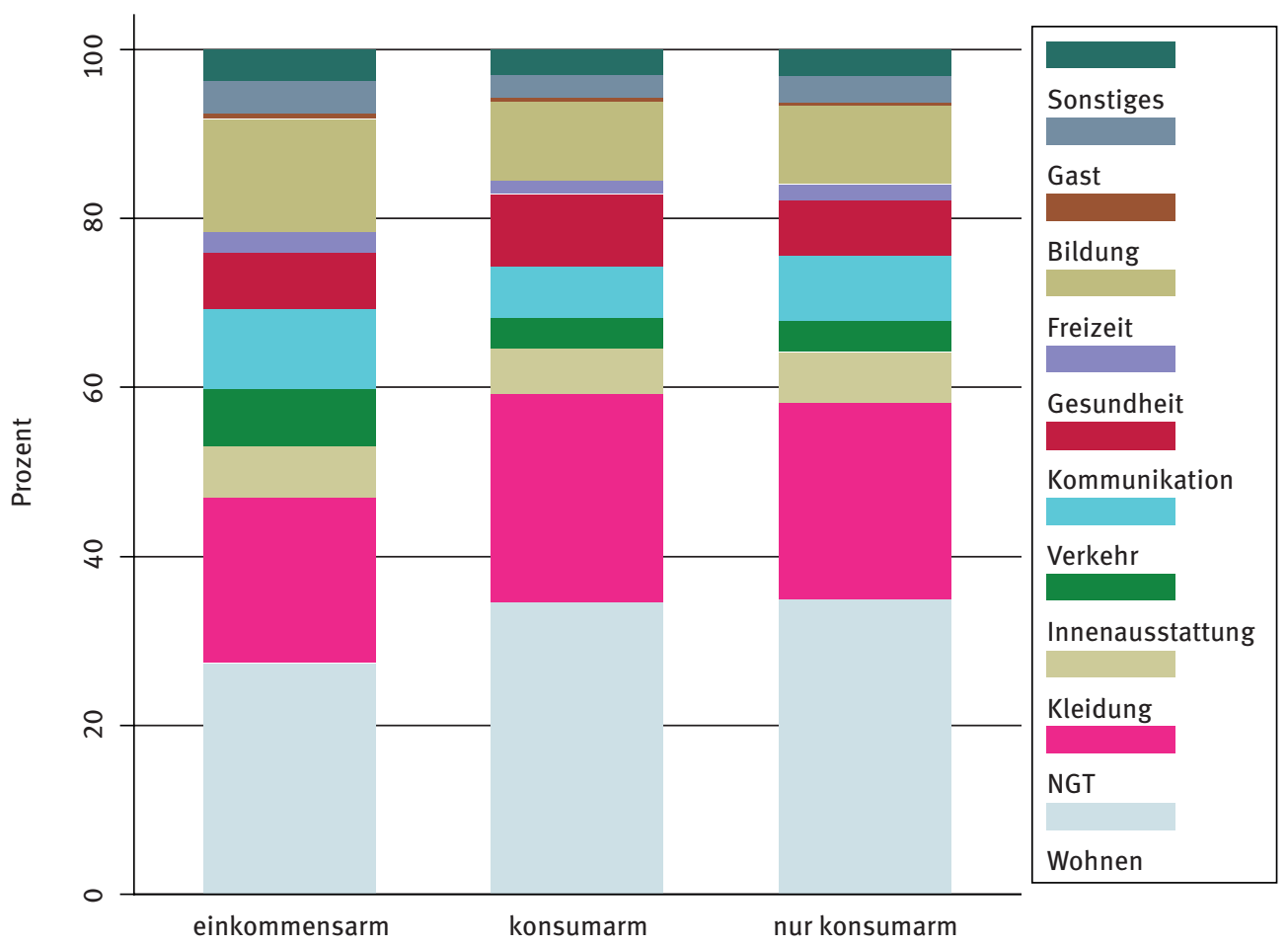

Abb. 2: Konsumstruktur nach Armutsstatus, Quelle: EVS 1978-2008; eigene Berechnungen.

ko mit der Verrentung und bei Arbeitslosigkeit signifikant steigt, zeigen sich für diese Gruppen bei den Nur-Konsumarmen keine signifikanten Effekte. Hinsichtlich des Geschlechts haben Frauen auf der einen Seite ein höheres Risiko einkommensarm zu sein als Männer, auf der anderen Seite aber ein geringeres Risiko zu den Nur-Konsumarmen zu zählen. Bei den Konsumarmen lassen sich keine signifikant unterschiedlichen Armutsrisiken für Männer und Frauen feststellen. Eine mögliche Ursache für die signifikant höheren Einkommensarmutsrisiken von Frauen kann die Lohndifferenz zwischen Männern und Frauen sein.

\section{Vergleich der Konsumstruktur}

Da der Ausgabenanteil für Wohnen und Nahrungsmittel im Sinne der Engel'schen und Schwabe'schen Gesetze häufig als Wohlstandsindikator verwendet wird, untersucht der folgende Abschnitt, wie sich die Konsumstruktur und die entsprechenden Budgetanteile je nach Armutsstatus unterscheiden. Dabei gilt: Je niedriger die Budgetanteile für Nahrung und Wohnen, desto höher der Wohlstand. Abbildung 2 stellt die Ausgabenprofile gegenüber.

Der Vergleich zwischen Einkommens- und Konsumarmen zeigt, dass Konsumarme, gemessen an den Aus- gabenanteilen für die Grundbedürfnisse, deutliche Wohlstandsverluste einstecken müssen. Ihre Ausgabenanteile für Wohnen und Nahrungsmittel liegen signifikant über denen Einkommensarmer. Stärkere Einsparungen werden von den Konsumarmen in den Bereichen Verkehr, Innenausstattung und Freizeitgestaltung vorgenommen. Die Ausgaben in den Bereichen Freizeit und Verkehr können dabei als solche sozialer Teilhabe interpretiert werden. Nur bei den Ausgaben für Kommunikation zeigen sich keine signifikanten Unterschiede. Die Verwendung eines ausgabenbasierten Wohlstandsindikators identifiziert somit stärker diejenigen Personen, die ihre Möglichkeiten der sozialen Teilhabe stark einschränken müssen, um ihr physisches Existenzminimum decken zu können.

Die Konsumstruktur Nur-Konsumarmer ist im Vergleich zu der der Einkommensarmen ebenfalls durch höhere Ausgabenanteile in den Bereichen Wohnen und Nahrungsmittel gekennzeichnet. Im Gegensatz zur gesamten Gruppe Konsumarmer investieren Nur-Konsumarme zusätzlich einen signifikant höheren Anteil ihrer Ausgaben in Bekleidung und Verkehr, wobei die Differenzen weniger als einen Prozentpunkt betragen. Bei den Ausgabenanteilen für Gaststätten- und Beherbergungsdienstleistungen finden sich keine signifikanten Unterschiede. Eine rein einkommensorientierte Wohlstandsanalyse würde somit eine Gruppe von Personen nicht berücksichtigen, die zu- 
Tab. 5: Konsumquote und Anteil Overspending getrennt nach Armutsstatus

\begin{tabular}{lrrr}
\hline & einkommensarm & konsumarm & nur-konsumarm \\
\hline$\emptyset$ monatliche Einkommen (in €) & 498 & 592 & 898 \\
$\emptyset$ monatliche Ausgaben (in €) & 504 & 385 & 408 \\
Konsumquote (in \%) & 108,9 & 75,9 & 48,8 \\
Anteil Overspending (in \%) & 25,7 & 6,9 & 0 \\
Höhe des Overspendings (in €) & 223 & 81 & - \\
Auflösung Vermögen bei Overspendern (in \%) & 93,2 & 94,4 & - \\
Aufnahme Kredite bei Overspendern (in \%) & 12,9 & 8,4 & - \\
Anteil verschuldeter Personen (in \%) & 25,2 & 30,9 & 47,5 \\
Höhe Verschuldung/Einkommen (in \%) & 27,7 & 41,3 & 77,5 \\
\hline
\end{tabular}

Quelle: EVS 1978-2008; eigene Berechnungen. Anmerkung: a= Spaltensummen von über $100 \%$ erklären sich dadurch, dass Personen ihre Ausgaben theoretisch über mehrere der genannten Optionen finanzieren können.

mindest laut ihres Konsumprofils deutlich benachteiligt ist.

\section{Finanzielle Situation}

In einem weiteren Schritt werden Aspekte der finanziellen Situation einkommens- und konsumarmer Haushalte miteinander verglichen (vgl. Tab. 5). Die Situation einkommensarmer Haushalte ist insbesondere durch hohe Ausgaben in Relation zum Einkommen gekennzeichnet. Ihre durchschnittliche Konsumquote liegt dementsprechend bei fast $110 \%$. Im Gegensatz dazu geben Konsumarme im Schnitt weniger als $80 \%$ ihrer Einnahmen aus. Ihr unterdurchschnittlicher Konsum ist somit nicht zwangsläufig durch zu geringe Einkommen bedingt. Dass die Konsumquote Nur-Konsumarmer unter der der Einkommens- und Konsumarmen liegt, ist eine logische Konsequenz der Definition. Allerdings überrascht der deutliche Abstand zu der Konsumquote Nicht-Armer: Während nicht-arme Haushalte im Schnitt 77\% ihrer verfügbaren Einkommen verkonsumieren, liegt dieser Anteil bei den Nur-Konsumarmen bei unter $50 \%$.

Wie der Vergleich der Konsumquoten bereits andeutet, tritt Overspending ${ }^{2}$ bei Konsumarmen deutlich seltener auf als bei Einkommensarmen: Knapp 7 \% der Konsumarmen gehören zu den Overspendern, bei den Einkommensarmen sind es mit knapp $26 \%$ fast viermal so viele. Dieser hohe Anteil an Overspendern bei den Einkommensarmen

2 Da die Angaben zu Einnahmen und Ausgaben in der EVS unter Ungenauigkeiten in der Anschreibung leiden, kann es vorkommen, dass das Vorkommen von Overspending überschätzt wird. Um auf diese messbedingten Fehler zu kontrollieren, wird im Folgenden nur in den Fällen von Overspending gesprochen, in denen die Ausgaben die Einnahmen um mehr als das 1,1fache übertreffen. kann als Erklärung für den früheren Befund herangezogen werden, dass sich Einkommensarme in der Konsumperspektive besserstellen. Einkommensarme tätigen über die Mobilisierung finanzieller Ressourcen zusätzlich zu den regulären Einkommen durchschnittlich höhere Ausgaben und erzielen daher eine höhere relative Konsumposition. Die absolute Differenz zwischen Einnahmen und Ausgaben ist bei den einkommensarmen Overspendern zudem deutlich höher. Die nur-konsumarmen Personen geben hingegen nicht mehr aus als sie einnehmen.

Das angesprochene Overspending muss nicht zwangsläufig ein Merkmal für finanzielle oder materielle Notlagen darstellen. Einen Hinweis darauf, inwiefern Overspending tatsächlich mit finanzieller Not verbunden ist, kann ein Blick auf die Finanzierung des Overspendings der Einkommens- und Konsumarmen liefern. Können die überschüssigen Ausgaben anhand von kurzfristig liquidierbaren Ressourcen, wie z. B. Gelder auf Spar- oder Girokonten, getilgt werden, dann sollte diese Person mit einer geringeren Wahrscheinlichkeit unter ein Armutsverständnis fallen als eine Person, die dafür einen Kredit aufnehmen müsste. Tabelle 5 zeigt daher die jeweiligen Finanzierungsquellen des Overspendings. Sowohl bei den Einkommens- als auch bei den Konsumarmen wird der Großteil des Overspendings über die Auflösung von Vermögen finanziert. Überwiegend handelt es sich dabei um Geldvermögen; Sachvermögen wird nur in äußerst seltenen Fällen liquidiert. Der Anteil des durch Vermögensauflösung finanzierten Overspendings ist bei den Konsumarmen etwas höher. Einkommensarme müssen hingegen etwas häufiger auf Kredite zurückgreifen. Die Analyse der Relationen von Einnahmen und Ausgaben bei den Einkommens- und Konsumarmen liefert im Gegensatz zum Vergleich der Konsumstrukturen somit keinen Hinweis darauf, dass durch die Fokussierung auf die Einkommen 
Tab. 6: Ausstattungsgrad nach Armutsstatus

\begin{tabular}{lrrr}
\hline & einkommensarm & konsumarm & nur-konsumarm \\
\hline Ausstattungsgrad (in \%) & 44,4 & 44,1 & 50,0 \\
Wohnfläche pro Person (in $\mathbf{~ m}^{2}$ ) & 41 & 37 & 37 \\
Hausbesitzer/Wohnungseigentümer (in \%) & 17,0 & 24,3 & 50,3 \\
\hline
\end{tabular}

Quelle: EVS 1978-2008; eigene Berechnungen.

eine Gruppe besonders benachteiligter Haushalte unberücksichtigt bleibt.

Die Angaben zum Overspending und der Konsumquote beruhen jedoch nur auf Informationen über die Ausgaben und Einnahmen während des Anschreibungszeitraums. Betrachtet man hingegen den Anteil an Haushalten mit Hypotheken- und Konsumentenkreditrestschulden, dann sind die Konsumarmen und insbesondere die Nur-Konsumarmen deutlich häufiger betroffen. Fast die Hälfte aller Nur-Konsumarmen ist verschuldet, bei den Einkommensarmen ist es nur knapp ein Viertel. Der Grad der Verschuldung ist bei den Nur-Konsumarmen ebenfalls deutlich höher; sie haben Schulden, die knapp 80 \% ihres Jahreseinkommens entsprechen. Bei den Einkommensarmen beträgt der Anteil etwas unter 30 \%. Die niedrige Konsumquote Nur-Konsumarmer kann somit auch eine Folge hoher Kreditrückzahlungen sein.

\section{Ausstattung mit langlebigen Gebrauchsgü- tern und Wohnsituation}

Abschließend werden Einkommens- und Konsumarme sowie Nur-Konsumarme hinsichtlich der Ausstattung ihres Haushalts und der Wohnsituation verglichen (vgl. Tab. 6). Betrachtet wird dabei der Ausstattungsgrad, d.h. wie viel Prozent der erhobenen langlebigen Gebrauchsgüter im Haushalt vorhanden sind. Informationen über den Grund des Fehlens, z. B. mangelnde finanzielle Ressourcen oder persönliche Präferenzen, stellt die EVS nicht bereit. Die Ausstattungsgrade einkommens- und konsumarmer Haushalte sind nahezu identisch und liegen jeweils bei ca. $44 \%$. Die Haushalte Nur-Konsumarmer sind hingegen besser ausgestattet und verfügen über die Hälfte der erhobenen Gebrauchsgüter. Da die Gründe für das Nichtvorhandensein der Güter nicht bekannt sind, können auf der Grundlage dieser Zahlen allerdings nur eingeschränkt Rückschlüsse auf einen schlechteren Lebensstandard gezogen werden.

Als weiterer Indikator des Lebensstandards wurde die Wohnfläche pro Person betrachtet. In einkommensarmen Haushalten standen jeder Person durchschnittlich $41 \mathrm{~m}^{2}$ zur Verfügung. Mit $37 \mathrm{~m}^{2}$ ist die Fläche bei konsumarmen Personen wie auch bei sogenannten Nur-Konsumarmen deutlich geringer. Dafür sind mehr Konsumarme Hausoder Wohnungseigentümer: Während Einkommensarme nur in $17 \%$ der Fälle über Wohneigentum verfügen, sind es bei den Konsumarmen $24 \%$ und bei den Nur-Konsumarmen sogar $50 \%$. Hypotheken oder Baudarlehen könnten somit den vorherigen Befund einer relativ hohen Anzahl verschuldeter Haushalte unter den Konsumarmen erklären.

\section{Zusammenfassung und Diskussion}

Die Verwendung von Konsum als Wohlstandsindikator ist in Deutschland wenig verbreitet. Die im Rahmen dieses Artikels durchgeführten Analysen mit der EVS der Jahre 1978 bis 2008 sollen dazu beitragen, bisherige Erkenntnisse aus der vornehmlich einkommensbasierten deutschen Ungleichheits- und Armutsforschung zu erweitern.

Dass Einkommen und Konsumausgaben tatsächlich zwei unterschiedliche Aspekte von Wohlstand messen, zeigen unter anderem die auf der jeweiligen Grundlage ermittelten Ungleichheits- und Armutsmaße. Zwar hat die Ungleichheit beider Indikatoren im Zeitverlauf zugenommen, allerdings sind die Konsumausgaben weniger ungleich verteilt als die Einkommen. Zudem kann die für die Einkommen festgestellte Polarisierung bei den Konsumausgaben nur in einer abgeschwächten Form nachgewiesen werden. Ein Vergleich der jeweiligen relativen Einkommens- und Konsumpositionen zeigt ferner, dass die Mehrheit der betrachteten Personen bei einer ausgabenbasierten Betrachtung besser dasteht. Relativ niedrige Einkommen scheinen somit zumindest auf kurze Sicht den Lebensstandard nicht negativ zu beeinflussen. Nicht nur die konsumbasierten Ungleichheitsmaße sprechen für einen weniger dramatischen Wohlstandsverlust in Deutschland, auch die verschiedenen Armutsmaße sind mit Ausnahme des ersten Beobachtungsjahrs stets niedriger, wenn die Konsumausgaben als Wohlstandsindika- 
tor verwendet werden. Auch die Armutsintensität ist in diesem Fall weniger stark ausgeprägt. Die präsentierten Analysen beschränken sich aus methodischen Gründen auf westdeutsche Haushalte mit einem deutschen Haushaltsvorstand. Hinsichtlich der ermittelten Armuts- und Ungleichheitsmaße ist davon auszugehen, dass diese tendenziell zu niedrig ausfallen, da für Personen mit Migrationshintergrund sowie für Ostdeutsche - zumindest gemessen am Einkommen - bereits deutlich höhere Armutsgefährdungsquoten nachgewiesen werden konnten als für (West-)Deutsche (Statistisches Bundesamt 2013, 2014). Inwiefern die Ergebnisse des Vergleichs der Lebenslagen Einkommens- und Konsumarmer generalisierbar sind, müssen weitere Studien zeigen.

Die von beiden Indikatoren definierten Armutspopulationen überschneiden sich nur teilweise, wobei der Anteil an Personen, die aus der Sicht beider Indikatoren als arm bezeichnet werden können, im Zeitverlauf zugenommen hat. Es gibt somit eine Gruppe konsumarmer Personen, die bei einer rein einkommensbasierten Betrachtung unberücksichtigt bliebe, die sogenannten „Nur-Konsumarme“. Hinsichtlich soziodemografischer Merkmale gibt es allerdings kaum Unterschiede, die die Differenz zwischen Einkommens- und Konsumarmut erklären könnten. Ein generell höheres Armutsrisiko haben junge Haushalte und Alleinstehende. Bei den Einkommensarmen trifft dies zudem auf Frauen zu, was sich möglicherweise durch geschlechtsspezifische Lohndifferenzen erklären lässt.

In einem weiteren Schritt wurde daher die Vermögenssituation der verschiedenen Armutspopulationen verglichen. Aus dieser Perspektive zeigt sich, dass die Gruppe der Nur-Konsumarmen aus einem verhältnismäßig hohen Anteil verschuldeter Personen besteht. Der hohe Schuldneranteil bedingt möglicherweise die geringen Konsumausgaben dieser Gruppe, denn der Vergleich der Konsumquoten zeigt, dass die geringen Konsumausgaben der Nur-Konsumarmen nicht zwangsläufig die Folge fehlender finanzieller Ressourcen sind. Die Nur-Konsumarmen geben im Durchschnitt ca. die Hälfte ihrer Einkommen für den Konsum aus, in keinem Fall überschreiten die Ausgaben die Einnahmen. Allerdings sind fast $80 \%$ der Nur-Konsumarmen verschuldet und müssen einen großen Anteil ihrer Einkommen für die Tilgung und Verzinsung von Krediten aufwenden, welche in der EVS-Klassifikation nicht zu den Konsumausgaben gezählt werden. Der hohe Schuldneranteil dieser Gruppe kann wiederum zum Teil durch den relativ hohen Anteil an Wohneigentümern erklärt werden.

Durch die zusätzliche Betrachtung der Konsumausgaben wird somit der Blick auf eine Gruppe von Personen gelenkt, die aufgrund von Schuldentilgungen ihren Kon- sum (kurzfristig) einschränken muss. Je nach Art der Investition, wie z. B. im Falle von Wohneigentum, können sie jedoch langfristig von deren Nutzen profitieren und ihr Lebensstandard kann trotz punktuell geringer Ausgaben als relativ hoch eingeschätzt werden. Zudem ist davon auszugehen, dass sie - insofern keine unerwarteten Einkommensbußen auftreten - sich selbst aus ihrer Armutslage befreien können. Allerdings zeigen die Analysen auch, dass die Nur-Konsumarmen im Vergleich zu den Einkommensarmen höhere Ausgabenanteile in den Bereichen Wohnen und Nahrungsmittel verzeichnen. Es verbleibt somit ein geringer finanzieller Spielraum für die soziale Teilhabe, deren Sicherung wiederum ein zentrales Ziel des deutschen Sozialstaats ist. Für diese Gruppe besteht zudem aufgrund der in Relation zum Einkommen sehr hohen Verschuldung die Gefahr, durch unerwartete negative Lebensereignisse, wie z. B. Arbeitslosigkeit oder familiäre Veränderungen, in die existenziell bedrohliche Armut abzurutschen. Hinsichtlich der Frage nach den sozialen Folgen von Konsumarmut kann zumindest für die verschuldeten Konsumarmen auf Forschungsergebnisse verwiesen werden, nach denen verschuldete Personen neben den wirtschaftlichen Folgen unter Stigmatisierung und sozialer Exklusion leiden (Bundesministerium für Familie, Senioren, Frauen und Jugend 2008; Maischatz 2014). Wohlstandsmessungen allein auf der Basis von Konsumausgaben würden somit zu verzerrten Aussagen verleiten, da die Problematik der Verschuldung unberücksichtigt bleibt.

Die Analysen lassen nicht erkennen, warum die knapp über $20 \%$ der Nur-Konsumarmen ohne Kreditschulden ihren Konsum einschränken. Mögliche Erklärungen sind eine hohe Sparneigung aus Vorsichts- oder Vorsorgemotiven und ein hoher Ausstattungsgrad. Diese Personen würden auch bei einer um den Verschuldungsaspekt erweiterten Einkommensarmutsmessung nicht berücksichtigt.

Insgesamt kann festgehalten werden, dass sich das Lebenslagenprofil der Nur-Konsumarmen deutlich von dem der Einkommensarmen unterscheidet und viele Indikatoren eine Beeinträchtigung in materiellen und sozialen Bereichen indizieren. Ausreichende finanzielle Ressourcen sind demnach eine notwendige, aber keine hinreichende Bedingung zur Vermeidung von Armut. Darüber hinaus unterscheiden sich auch die Nur-Konsumarmen zum Teil deutlich hinsichtlich ihrer finanziellen Rahmenbedingungen, und es können verschiedene Motive für die geringen Konsumausgaben unterstellt werden. Für die Einschätzung des Mehrgewinns der Armutsmessung durch die Konsumbetrachtung wären allgemein und insbesondere für die Gruppe der nicht verschuldeten NurKonsumarmen Informationen über die subjektive Bewer- 
tung des Konsumniveaus hilfreich, welche die EVS jedoch nicht bereitstellt.

Zur Ableitung konkreter sozialpolitischer Implikationen bedarf es indes weiterer differenzierterer Analysen, die die im Rahmen dieser Arbeit aufgeworfenen Forschungsfragen näher beleuchten. Die Ergebnisse legen allerdings die Vermutung nahe, dass sich die Bedarfe der Einkommens- und Konsumarmen zu deutlich unterscheiden, als dass eine einheitliche sozialpolitische Maßnahme den Anforderungen gerecht werden könnte.

\section{Literatur}

Aguiar, M. \& M. Bils, 2011: Has Consumption Inequality Mirrored Income Inequality? NBER Working Paper 16807.

Anand, S., 2001: The Measurement of Inequality. S. 81-105 in: S. Subramanian (Hrsg.): Measurement of Inequality and Poverty. New Delhi: Oxford University Press.

Attanasio, O., E. Hurst \& L. Pistaferri, 2012: The Evolution of Income, Consumption, and Leisure Inequality in the US, 1980-2010. NBER Working Paper 17982.

Baudrillard, J., 1988: The System of Objects. Oxford: Polity Press.

Beck, U., 1983: Jenseits von Klasse und Stand? Soziale Ungleichheiten, gesellschaftliche Individualisierungsprozesse und die Entstehung neuer sozialer Formationen und Identitäten. S. 35-74 in: R. Kreckel (Hrsg.): Soziale Ungleichheiten (Soziale Welt Sonderband 2). Göttingen: Schwartz.

Becker, I. \& R. Hauser, 1994: Die Entwicklung der Einkommensverteilung in der Bundesrepublik Deutschland in den siebziger und achtziger Jahren. Arbeitspapier Nr. 3. August 1994. Frankfurt am Main: Johann Wolfgang Goethe-Universität.

Becker, I. \& R. Hauser, 2004: Lebenslagen in Deutschland. Armutsund Reichtumsberichterstattung der Bundesregierung. Forschungsprojekt Verteilung der Einkommen 1999-2003. Bonn: Bundesministerium für Gesundheit und Soziale Sicherung.

Becker, I. \& R. Schüssler, 2014: Das Grundsicherungsniveau: Ergebnis der Verteilungsentwicklung und normativer Setzungen. Arbeitspapier 298. Düsseldorf: Hans-BöcklerStiftung.

Berner, E., 2015: Unfassbare Armut. Definitionsprobleme und politische Brisanz. S.1-10 in: M. Boatca, K. Fischer \& G. Hauck (Hrsg.): Handbuch Entwicklungsforschung. Wiesbaden: Springer VS.

Blundell, R., L. Pistaferri \& I. Preston, 2008: Consumption Inequality and Partial Insurance. American Economic Review 98(5): 1887-921.

Bögenhold, D. \& U. Fachinger, 2000: The Social Embeddedness of Consumption: Towards the Relationship of Income and Expenditures over Time in Germany. MPRA Paper No 1128.

Bögenhold, D. \& U. Fachinger, 2005: Konsummuster im Kontrast: Die Entwicklung unterschiedlicher Einkommensverwendungsmuster anhand verschiedener Haushaltstypen im zeitlichen Wandel. MPRA Working Paper No. 1124.
Bourdieu, P., 1987: Die feinen Unterschiede. Kritik der gesellschaftlichen Urteilskraft, Frankfurt am Main: Suhrkamp.

Brewer, M., A. Goodman \& A. Leicester, 2006: Household Spending in Britain. What Can it Teach Us About Poverty? Bristol: The Policy Press.

Brzozowski, M. \& T.F. Crossley, 2011: Viewpoint: Measuring the well-being of the poor with income or consumption: a Canadian perspective. Canadian Journal of Economics 44(1): 88-106.

Bundesministerium für Familie, Senioren, Frauen und Jugend, 2008: Lebenslagen von Familien und Kindern. Überschuldung privater Haushalte. Expertise zur Erarbeitung des dritten Armuts- und Reichtumsberichtes der Bundesregierung, Nr. 22/2008.

Christoph, B., J. Pauser \& J. Wiemers, 2014: Konsummuster und Konsumarmut von SGB-II-Leistungsempfängern. IAB-Discussion Paper 9/2014. Nürnberg: Institut für Arbeitsmarkt und Berufsforschung.

Cowell, F. \& M. P. Victoria-Feser, 1996: Robustness Properties of Inequality Measures. Econometrica 64(1): 77-101.

Cutler, D.M. \& L.F. Katz, 1992: Rising Inequality? Changes in the Distribution of Income and Consumption in the 1980's. Trends in Nonwage Inequality 82(2): 546-51.

Deutscher Paritätischer Wohlfahrtsverband, 2015: Die zerklüftete Republik. Bericht zur regionalen Armutsentwicklung in Deutschland 2014. Berlin: Paritätischer Gesamtverband.

Foster, J., J. Greer \& E. Thorbecke, 1984: A Class of Decomposable Poverty Measures. Econometrica 52(3): 761-66.

Friedman, M., 1957: A Theory of the Consumption Function. Princeton: Princeton University Press.

Grabka, M. M. \& J. R. Frick, 2010: Weiterhin hohes Armutsrisiko in Deutschland: Kinder und junge Erwachsene sind besonders betroffen. Berlin: DIW.

Grabka, M.M., J. Goebel \& J. Schupp, 2012: Höhepunkte der Einkommensungleichheit in Deutschland überschritten? DIW-Wochenbericht 79(43): 3-15.

Grauel, J., 2010: Soziale Differenzierung durch moralischen Konsum? Vortrag auf dem Werkstattgespräch „Konsummuster: Differenzierung und Ungleichheit" am Soziologischen Forschungsinstitut (SOFI) an der Universität Göttingen am 19.2.2010

Groh-Samberg, O. \& J. Goebel, 2007: Armutsmessung im Zeitverlauf. Wirtschaftsdienst 6/2007: 397-403.

Gross, P., 1994: Die Multioptionsgesellschaft. Frankfurt am Main: Suhrkamp.

Hagenaars, A., K. de Vos \& A. Zaidi, 1994: Poverty Statistics in the late 1980s: Research Based on Micro-data. Office for Official Publications of the European Communuities. Luxembourg.

Haughton, J. \& S.R. Khandker, 2009: Handbook of Poverty and Inequality. Washington: The World Bank.

Hradil, S. 1987: Sozialstrukturanalyse in einer fortgeschrittenen Gesellschaft, Opladen: Leske + Budrich.

Johnson, D. \& S. Shipp, 1997: Trends in Inequality Using Consumption-Expenditures: The U.S. from 1960 to 1993. Review of Income and Wealth 43(2): 133-52.

Johnson, D. \& T.M. Smeeding, 1998: Measuring the Trends in Inequality of Individuals and Families: Income and Consumption, BLS Manuscript.

Keynes, J.M., 1937: The General Theory of Employment. The Quarterly Journal of Economics 51(2): 209-223.

Klocke, A., 2000: Methoden der Armutsmessung. Zeitschrift für Soziologie 29(4): 313-29. 
Krueger, D. \& F. Perri, 2006: Does Income Inequality Lead to Consumption Inequality? Evidence and Theory. Review of Economic Studies, 73: 163-93.

Lüthi, A., 1981: Messung wirtschaftlicher Ungleichheit. Berlin/ Heidelberg: Springer.

Maischatz, K. (2014). Soziale Beziehungen unter Exklusionsbedingungen - Zum Zusammenhang von Überschuldung, Verbraucherinsolvenz und Sozialkapital. Lüneburg: Dissertation.

McGregor, P.P.L. \& V.K. Barooah, 1992: Is Low-Spending or Low Income a Better Indicator of Whether or Not a Household is Poor: Some Results From the 1985 Family Expenditure Survey. Journal of Social Policy 21(1): 53-69.

Meyer, B.D. \& J.X. Sullivan, 2003: Measuring the Well-Being of the Poor Using Income and Consumption. Journal of Human Resources 38: 1180-220.

Meyer, B.D. \& J.X. Sullivan, 2004: The Effects of Welfare and Tax Reform: the Material Well-Being of Single Mothers in the 1980s and 1990s. Journal of Public Economic 88: 1387-1420.

Meyer, B.D. \& J.X. Sullivan, 2011: Consumption and Income Poverty over the Business Cycle. NBER Working Papers 16751.

Modigliani, F. \& R. Brumberg, 1954: Utility Analysis and the Consumption Function: an Interpretation of Cross-Section Data. S. 128-97 in: K. K. Kurihara (Hrsg.): The Collected Papers of Franco Modigliani. Vol. 2: The Life Cycle Hypothesis of Saving, Cambridge MA.: MIT Press.

Neurath, 0.1981: Gesammelte philosophische und methodische Schriften - Band 1. Wien: Hölder-Pichler-Tempsky.

Noll, H.-H., 2007: Household Consumption, Household Incomes and Living Standards. A review of related recent research activities, Mannheim.

Noll, H.-H. \& S. Weick, 2005a: Relative Armut und Konzentration der Einkommen deutlich gestiegen. Informationsdienst Soziale Indikatoren (ISI) 33: 1-6.

Noll, H.-H. \& S. Weick, 2005b: Markante Unterschiede in den Verbrauchsstrukturen verschiedener Einkommenspositionen trotz Konvergenz: Analysen zu Ungleichheit und Strukturwandel des Konsums. Informationsdienst Soziale Indikatoren (ISI) 34: 1-5.

Noll, H.-H. \& S. Weick, 2007: Einkommensarmut und Konsumarmut - unterschiedliche Perspektiven und Diagnosen. Informationsdienst Soziale Indikatoren (ISI) 37: 1-6.

Poterba, J.M., 1991: Is the Gasoline Tax Regressive? S. 145-164 in: D. Bradford (Hrsg.): Tax Policy and the Economy. New York: MIT Press.

Sabelhaus, J. \& U. Schneider, 1997: Measuring The Distribution Of Well-Being: Why Income and Consumption Give Different Answers. Discussion Paper 201. Washington: Urban Institute.

Scheuch, E. K., 1975: Der Charakter des Konsums in modernen Industriegesellschaften - ein Beitrag zur Soziologie des Konsums. Hamburger Jahrbuch für Wirtschafts- und Gesellschaftspolitik: 111-28.

Schneider, N. F., 2000: Konsum und Gesellschaft. S. 9-22 in: D. Rosenkranz \& N. F. Schneider (Hrsg.): Konsum. Opladen: Leske + Budrich.

Sen, A., 1976: Poverty. An Ordinal Approach to Measurement. Econometrica 44(2): 219-231.

Sierminska, E. \& T.I. Garner, 2003: A Comparison of Income, Expenditures, and Home Market Value Distributions Using Luxembourg Income Study Data From the 1990’S. Study
Working Paper No. 338. Luxemburg: Institute of SocioEconomic Research.

Simmel, G., 1900: Philosophie des Geldes. Leipzig: Duncker \& Humblot.

Simmel, G., 1905: Philosophie der Mode. In: H. Landsberg (Hrsg.): Reihe Moderne Zeitfragen, Berlin: Pan-Verlag o. J.

Slater, D., 1997: Consumer Culture and Modernity. Cambridge: Polity Press.

Slesnick, D.T., 1993: Gaining Ground: Poverty in the Postwar United States. Journal of Political Economy 101(1): 1-38.

Townsend, P., 1979: Poverty in the United Kingdom. Harmondsworth: Penguin.

Veblen, T. B., 1899: Theory of the Leisure Class. An Economic Study in the Evolution of Institutions. New York: Macmillan.

Zaidi, M.A. \& K. de Vos, 2001: Trends in Consumption-Based Poverty and Inequality in the European Union during the 1980s. Journal of Population Economics 14: 367-90.

Zapf, W., S. Breuer, J. Hampel, P. Krause, H.-M. Mohr \& E. Wiegand, 1987: Individualisierung und Sicherheit. Untersuchungen zur Lebensqualität in der Bundesrepublik Deutschland. München: Beck.

Zusatzmaterial: Die Onlineversion dieses Artikels (DOI: 10.1515/zfsoz-2015-1011) bietet Zusatzmaterial für autorisierte Benutzer.

\section{Autorenvorstellung}

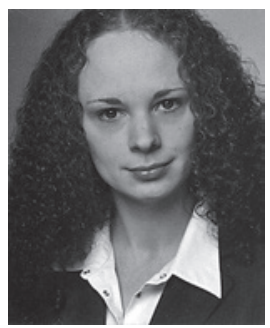

\section{Katharina Hörstermann}

Institut für Soziologie und Sozialpsychologie, Universität zu Köln, AlbertusMagnus-Platz, 50923 Köln, E-Mail: hoerstermann@wiso.uni-koeln.de

Katharina Hörstermann, geb. 1984 in Lindlar, Studium der Sozialwissenschaften an der Universität zu Köln. Seit Ende 2011 Promotionsstudent an der Universität zu Köln. Von 2011 bis 2015 wissenschaftl. Mitarbeiterin im DFG-Forschungsprojekt „Konsum und Lebensstandard - Eine Langfristanalyse der Einkommensverwendung in Deutschland“.

Forschungsschwerpunkte: Konsum, Lebensstandard, soziokulturelles Existenzminimum

Wichtigste Publikationen: „Wer nicht arbeitet, soll auch nicht essen!“ Eine Vignettenanalyse zur Bestimmung eines Einkommensmindestbedarfs. Zeitschrift für Sozialreform 61(2) 2015: 171-199 (mit Hans-Jürgen Andreß); Lebensstandard und Deprivation im Alter in Deutschland. Stand und Entwicklungsperspektiven. Zeitschrift für Sozialreform 58(2) 2012: 209-235 (mit Hans-Jürgen Andreß). 